\title{
IRRATIONALITY OF MOTIVIC ZETA FUNCTIONS
}

\author{
MICHAEL J. LARSEN AND VALERY A. LUNTS
}

\begin{abstract}
Let $K_{0}\left(\operatorname{Var}_{\mathbb{Q}}\right)[1 / \mathrm{E}]$ denote the Grothendieck ring of $\mathbb{Q}$-varieties with the Lefschetz class inverted. We show that there exists a K3 surface $X$ over $\mathbb{Q}$ such that the motivic zeta function $\zeta_{X}(t):=\sum_{n}\left[\operatorname{Sym}^{n} X\right] t^{n}$ regarded as an element in $K_{0}\left(\operatorname{Var}_{\mathbb{Q}}\right)[1 / \mathrm{E}][[t]]$ is not a rational function in $t$, thus disproving a conjecture of Denef and Loeser.
\end{abstract}

\section{INTRODUCTION}

Let $k$ be a field. We denote by $K_{0}\left(\operatorname{Var}_{k}\right)$ the Grothendieck group of $k$ varieties, i.e., the free abelian group generated by isomorphism classes of $k$-varieties modulo the cutting-and-pasting relations $[X]=[Y]+[X \backslash Y]$ for all pairs $(X, Y)$ consisting of a variety $X$ and a closed subvariety $Y$. It is endowed with a commutative ring structure characterized by $[X][Y]=$ $[X \times Y]$. (Note that we use variety to mean reduced separated scheme of finite type over $k$, but the Grothendieck ring would not be changed if we allowed non-reduced schemes or non-separated schemes, or limited ourselves to affine schemes.)

Following Kapranov [Ka], we define the motivic zeta function

$$
\zeta_{X}(t):=\sum_{n=0}^{\infty}\left[\operatorname{Sym}^{n} X\right] t^{n} \in K_{0}\left(\operatorname{Var}_{k}\right)[[t]],
$$

where $\operatorname{Sym}^{n} X$ is the symmetric $n$th power $X^{n} / \Sigma_{n}$.

By a motivic measure, we mean a homomorphism $\mu: K_{0}\left(\operatorname{Var}_{k}\right) \rightarrow A$, where $A$ is a commutative ring. We write $\mu\left(\zeta_{X}(t)\right)$ for the image of $\zeta_{X}(t)$ in $A[[t]]$. If $k$ is a finite field, $\mu:[X] \mapsto|X(k)|$ defines a motivic measure with values in $\mathbb{Z}$. The image $\mu\left(\zeta_{X}(t)\right) \in \mathbb{Z}[[t]]$ is the usual zeta function of $X$ and therefore rational as a function of $t$ by Dwork's theorem [Dw]. Kapranov asked [Ka, 1.3.5] whether this rationality holds for the motivic zeta function itself. He proved that this is so when $X$ is a curve with at least one $k$-point, even if $k$ is not a finite field. (Since $K_{0}\left(\operatorname{Var}_{k}\right)$ is not an integral domain $[\mathrm{PO}$, there is a question exactly what this means, which we settle for the purposes of this paper by saying that $\zeta_{X}(t)$ rational means that there exists a polynomial $B(t)=1+b_{1} t+\cdots+b_{n} t^{n}$ such that $B(t) \zeta_{X}(t) \in K_{0}\left(\operatorname{Var}_{k}\right)[t]$.)

In [LL], we proved that in general $\zeta_{X}(t)$ is not rational when $X$ is a surface. This does not quite finish the question, since for many purposes (especially motivic integration), the natural object to consider is not $K_{0}\left(\operatorname{Var}_{k}\right)$ but

ML was partially supported by NSF grant DMS-1702152. 
$K_{0}\left(\operatorname{Var}_{k}\right)[1 / \mathrm{E}]$, where $\mathrm{E}:=\left[\mathbb{A}^{1}\right]$. It is known $\mathrm{Bor}$ that $\mathrm{E}$ is a zero-divisor; see, also, Za , for an analysis of the annihilator of $\mathrm{E}$. One might still hope, therefore, that $\zeta_{X}(t)$ may be rational as a power series over $K_{0}\left(\operatorname{Var}_{k}\right)[1 / \mathrm{E}]$. No variant of the method of [LL can possibly test this, since the motivic measures constructed in that paper are birationally invariant and therefore vanish on $\mathrm{E}$. This made possible the conjecture of Denef and Loeser [DL, Conjecture 7.5.1] predicting that $\zeta_{X}(t)$ should satisfy this weaker rationality condition. In this paper, we show that in general it does not.

To explain our strategy, we begin by discussing certain motivic measures which cannot detect the irrationality of zeta functions. A reference for the following discussion is [LL2]. We endow $K_{0}\left(\operatorname{Var}_{k}\right)$ with the $\lambda$-structure in which the $[X] \rightarrow\left[\operatorname{Sym}^{n} X\right]$ operations play the role of symmetric powers; in other words, $\lambda^{n}([X])$ is defined to be the $t^{n}$ coefficient of $\zeta_{X}(t)^{-1}$. If $A$ is a finite $\lambda$-ring (in the sense that every element $a \in A$ can be written $a=b-c$ where $\lambda^{n} b=\lambda^{n} c=0$ for $n$ sufficiently large), then every $\lambda$-homomorphism $\mu: K_{0}\left(\operatorname{Var}_{k}\right) \rightarrow A$ is a motivic measure for which $\mu\left(\zeta_{X}(t)\right)$ is rational for all $X / k$.

Here is an example. Let $K\left(G_{k}, \mathbb{Q}_{l}\right)$ denote the Grothendieck ring of (virtual) finite-dimensional continuous representations of $G_{k}$, where, as usual, $0 \rightarrow V_{1} \rightarrow V_{2} \rightarrow V_{3} \rightarrow 0$ implies $\left[V_{2}\right]=\left[V_{1}\right]+\left[V_{3}\right]$. Then $K\left(G_{k}, \mathbb{Q}_{l}\right)$ is a $\lambda$-ring (even a special $\lambda$-ring), and

$$
[X] \mapsto \sum_{i=0}^{2 \operatorname{dim} X}(-1)^{i}\left[H^{i}\left(\bar{X}, \mathbb{Q}_{l}\right)\right],
$$

where $H^{i}\left(\bar{X}, \mathbb{Q}_{l}\right)$ denotes the $i$ th $l$-adic étale cohomology group of $\bar{X}$ as $G_{k^{-}}$ representation, defines a ring homomorphism $\mu$. It is a consequence of the Künneth formula and the isomorphism

$$
H^{i}\left(\operatorname{Sym}^{n} \bar{X}, \mathbb{Q}_{l}\right) \stackrel{\sim}{\rightarrow} H^{i}\left(\bar{X}^{n}, \mathbb{Q}_{l}\right)^{\Sigma_{n}}
$$

that $\mu$ is a $\lambda$-homomorphism. Thus $\mu\left(\zeta_{X}(t)\right)$ is rational in $t$ for all $X$, where the degree of numerator and denominator depend only on the dimension of the cohomology of $\bar{X}$.

In particular, if $X$ is a $\mathrm{K} 3$ surface, then $\mu\left(\zeta_{X}(t)\right)^{-1}$ is a polynomial of degree 24 , the product of a degree 22 polynomial corresponding to the $H^{2}$ term and the factors $(1-t)\left(1-\mu(\mathrm{E})^{2} t\right)$, corresponding to the $H^{0}$ and $H^{4}$ terms. We consider K3 surfaces of Picard number 18, in which the $H^{2}$ factor further decomposes $(1-\mu(\mathrm{E}) t)^{18} \Lambda(t)$.

We modify this construction in three ways. First, we consider coefficients in $\overline{\mathbb{F}}_{l}$ instead of $\mathbb{Q}_{l}$. Second, we use a modified Grothendieck ring $K_{l}^{\mathrm{sp}}\left(G_{k}\right)$ of Galois representations, in which we identify $\left[V_{2}\right]$ with $\left[V_{1}\right]+\left[V_{3}\right]$ only when $V_{2} \cong V_{1} \oplus V_{3}$ as $G_{k}$-modules. This is essential since the essence of our construction is to distinguish $\overline{\mathbb{F}}_{l}$-valued Galois representations which have the same semisimplification. Third, we replace $k$ by $k\left(\zeta_{l}\right)$ in order to trivialize the cyclotomic character $G_{k} \rightarrow \mathbb{F}_{l}^{\times}$(so that $€$ maps to 1.) Up to the 
$t^{l}$ coefficient, everything works as before, but the expression for $\mu\left(\zeta_{X}(t)\right)$ as rational function breaks down at the $t^{l}$ coefficient. No one $l$ value necessary excludes the possibility of rationality but by taking values of $l$ tending to infinity, we can prove that $\zeta_{X}(t)$ cannot be rational.

Assuming the characteristic of $k$ is 0 , we can define $\nu_{l}$ so that for every non-singular projective $k$-variety $X$, we have $\nu_{l}([X])=\left[H^{\bullet}\left(\bar{X}, \overline{\mathbb{F}}_{l}\right)\right]$ in the Grothendieck ring $K_{l}^{\mathrm{sp}}\left(G_{k\left(\zeta_{l}\right)}\right)$. It is easy to calculate the semisimplification of $H^{\bullet}\left(\overline{\operatorname{Sym}^{n} X}, \overline{\mathbb{F}}_{l}\right)$ as $G_{k\left(\zeta_{l}\right)}$-representation, but as $\operatorname{Sym}^{n} X$ is in general singular, we do not know when

$$
\nu_{l}\left(\left[\operatorname{Sym}^{n} X\right]\right)=\left[\operatorname{Sym}^{n} H^{\bullet}\left(\bar{X}, \overline{\mathbb{F}}_{l}\right)\right] .
$$

However, we show that this holds when all the cohomology of $X$ is in even degree and $l$ is sufficiently large compared to $n$. If $l$ is large compared to the degrees of the numerator and denominator of $\zeta_{X}(t)$, then the linear recurrence satisfied by the $\nu_{l}\left(\left[\operatorname{Sym}^{i} X\right]\right)$ ultimately implies that $\nu_{l}\left(\left[\operatorname{Sym}^{l} X\right]\right)$ is non-effective. This is a result of the breakdown of the correspondence between the $(\bmod l)$ representation theory of $\mathrm{SL}_{2}\left(\mathbb{F}_{l}\right)$ and the complex representation theory of $\mathrm{SL}_{2}(\mathbb{C})$ which occurs in dimension $l$.

Unfortunately, we do not know how to compute the value $\nu_{l}\left(\left[\operatorname{Sym}^{l} X\right]\right)$ directly, but using a generalization to arbitrary fields of Göttsche's relation [Go in $K_{0}\left(\operatorname{Var}_{k}\right)$ between the classes $\left[X^{[i]}\right]$ of the Hilbert schemes of $X$ and the classes of the symmetric powers of $X$, we can show that $\nu_{l}\left(\left[X^{[l]}\right]\right)$ is also non-effective. This is absurd, since $X^{[l]}$ is projective and non-singular.

In $\S 2$, we discuss Grothendieck groups of representations of finite groups, especially $\mathrm{SL}_{2}\left(\mathbb{F}_{l}\right)$ and $\mathrm{SL}_{2}\left(\mathbb{F}_{l}\right)^{2}$. In $\S 3$, we use the method of Bittner [Bi] to construct $\nu_{l}$. In $\S 4$, we show that there exists a $K 3$ surface over $\mathbb{Q}$ with the desired Galois-theoretic properties. The generalization of Göttsche's theorem to arbitrary base field is given in $\S 5$. In $\S 6$, we discuss some variants of the category of Chow motives which enable us to show that if $l$ is large compared to $n, \operatorname{Sym}^{n} X$ behaves like a non-singular variety as far as $\nu_{l}$ is concerned. The proof of the main theorem is in $\S 7$.

The referee of an earlier version of this paper called our attention to the preprint Bon of Mikhail Bondarko establishing that Joseph Ayoub's announced proof $\mathrm{Ay} 1, \mathrm{Ay} 2$ of the conservativity conjecture implies the rationality of the motivic zeta-function of any variety in characteristic 0 with values in the K-group of numerical motives. We would like to thank the referee for this and many other helpful suggestions.

We would like to gratefully acknowledge helpful conversations with Pierre Deligne, Vladimir Drinfeld, Lothar Göttsche, Luc Illusie, Mircea Mustaţă, and Geordie Williamson.

\section{Grothendieck Rings of Representations}

We fix an odd prime $l$ and an algebraic closure $\overline{\mathbb{F}}_{l}$ of the prime field $\mathbb{F}_{l}$, which we regard as a space with the discrete topology. For any topological 
group $G$, we denote by $K_{l}^{\mathrm{sp}}(G)$ the Grothendieck ring of the exact category given by split short exact sequences of finite dimensional continuous $\overline{\mathbb{F}}_{l}[G]$ modules.

We claim that, as an additive group, $K_{l}^{\mathrm{sp}}(G)$ is the free $\mathbb{Z}$-module on indecomposable continuous $\overline{\mathbb{F}}_{l}[G]$-modules. To see this, recall $[\mathrm{Kr}]$ that an additive category is Krull-Schmidt if every object is a finite direct sum of indecomposable objects whose endomorphism rings are local. As every finite-dimensional $G$-module has finite length, the category of such modules is Krull-Schmidt [ $\mathrm{Kr}, \S 5]$.

By the Krull-Remak-Schmidt theorem, this implies that the factors appearing in any decomposition into indecomposables, together with their multiplicities, are uniquely determined. We say an element of $K_{l}^{\mathrm{sp}}(G)$ is effective if it is a non-negative linear combination of indecomposable classes.

Any continuous homomorphism $G \rightarrow H$ induces a restriction homomorphism $K_{l}^{\mathrm{sp}}(H) \rightarrow K_{l}^{\mathrm{sp}}(G)$, which maps effective classes to effective classes. If $G \rightarrow H$ is surjective, then $\operatorname{Res}_{G}^{H}$ is injective because distinct indecomposable representations restrict to distinct indecomposable representations of $G$. A class in $K_{l}^{\mathrm{sp}}(H)$ is effective if and only if its restriction to $K_{l}^{\mathrm{sp}}(G)$ is so.

If $G$ is profinite, $K_{l}^{\mathrm{sp}}(G)$ is the direct limit of $K_{l}^{\mathrm{sp}}(G / H)$ as $H$ ranges over open normal subgroups of $G$. In this section, we consider only finite groups $G$ endowed with the discrete topology, so the continuity condition will play no role.

If $V$ is a $G$-representation, we define $\zeta_{V}(t) \in K_{l}^{\text {sp }}(G)[[t]]$ as follows:

$$
\zeta_{V}(t)=\sum_{n=0}^{\infty}\left[\operatorname{Sym}^{n} V\right] t^{n},
$$

where $\operatorname{Sym}^{n} V$ denotes the space of $\Sigma_{n}$-coinvariants of the tensor product $V^{\otimes n}$. Note that the notation makes sense only if $G$ is given.

Proposition 2.1. If $H_{1}$ and $H_{2}$ are finite groups, external tensor product defines an injective homomorphism $T: K_{l}^{\mathrm{sp}}\left(H_{1}\right) \otimes K_{l}^{\mathrm{sp}}\left(H_{2}\right) \rightarrow K_{l}^{\mathrm{sp}}\left(H_{1} \times H_{2}\right)$.

Proof. We need to show that if $\rho_{1}: H_{1} \rightarrow \mathrm{GL}\left(V_{1}\right)$ and $\rho_{2}: H_{2} \rightarrow \mathrm{GL}\left(V_{2}\right)$ are indecomposable representations, then $\rho_{12}: H_{1} \times H_{2} \rightarrow \operatorname{GL}\left(V_{1} \otimes V_{2}\right)$ is an indecomposable representation of $H_{1} \times H_{2}$ and that moreover, the isomorphism class of the representation $V_{1} \otimes V_{2}$ determines the isomorphism classes of $V_{1}$ and $V_{2}$. The second claim follows immediately by applying Krull-Remak-Schmidt to the restriction of $V_{1} \otimes V_{2}$ to $G_{1} \times\{1\}$ and $\{1\} \times G_{2}$.

To prove that $\rho_{12}$ is indecomposable, it suffices to prove that the centralizer $Z_{12}$ of the $\overline{\mathbb{F}}_{l}$-span of $\rho_{12}\left(H_{1} \times H_{2}\right)$ in $\operatorname{End}\left(V_{1} \otimes V_{2}\right)$ is a local $\overline{\mathbb{F}}_{l}$-algebra. To commute with $\rho_{12}\left(H_{1} \times H_{2}\right)$ is the same as to commute with $\rho_{12}\left(H_{1} \times\{1\}\right)$ and $\rho_{12}\left(\{1\} \times H_{2}\right)$. If $Z_{i}$ denotes the centralizer of $\rho_{i}\left(H_{i}\right)$ in $\operatorname{End}\left(V_{i}\right)$, and $Z_{i}^{\prime}$ is any $\overline{\mathbb{F}}_{l}$-linear complement of $Z_{i}$ in $\operatorname{End}\left(V_{i}\right)$, then the centralizer of 
$\rho_{12}\left(H_{1} \times\{1\}\right)$ is

$$
Z_{1} \otimes \operatorname{End}\left(V_{2}\right)=Z_{1} \otimes\left(Z_{2} \oplus Z_{2}^{\prime}\right)=Z_{1} \otimes Z_{2} \oplus Z_{1} \otimes Z_{2}^{\prime},
$$

the centralizer of $\rho_{12}\left(\{1\} \times H_{2}\right)$ is

$$
\operatorname{End}\left(V_{1}\right) \times Z_{2}=\left(Z_{1} \oplus Z_{1}^{\prime}\right) \otimes Z_{2}=Z_{1} \otimes Z_{2} \oplus Z_{1}^{\prime} \otimes Z_{2},
$$

and the intersection of these two centralizers is $Z_{1} \otimes Z_{2}$.

Each finite-dimensional representation is indecomposable if and only if its endomorphism ring is local [ $\mathrm{Kr}$, Proposition 5.4]. The tensor product of finite-dimensional local algebras over an algebraically closed field is again local [Law, Theorem 4], and this proves the proposition.

We will eventually be interested in the case $G=\mathrm{SL}_{2}\left(\mathbb{F}_{l}\right)^{2}$, but we start with $H=\mathrm{SL}_{2}\left(\mathbb{F}_{l}\right)$. We denote by $V_{i}$ the $i$ th symmetric power of the natural 2-dimensional $\overline{\mathbb{F}}_{l}$-representation of $H$ and by $\mathbf{W}$ the representation $V_{1} \otimes V_{l-1}$.

Proposition 2.2. We define

$$
F_{n} K_{l}^{\mathrm{sp}}(H)= \begin{cases}\operatorname{Span}_{\mathbb{Z}}\left(\left[V_{0}\right], \ldots,\left[V_{n}\right]\right) & \text { if } n \leq l-1, \\ \operatorname{Span}_{\mathbb{Z}}\left(\left[V_{0}\right], \ldots,\left[V_{l-1}\right],[\mathbf{W}]\right) & \text { if } n=l, \\ K_{l}^{\mathrm{sp}}(H) & \text { if } n>l .\end{cases}
$$

We have the following facts:

(1) The representation $\mathbf{W}$ is indecomposable.

(2) The product on $K_{l}^{\mathrm{sp}}(H)$ is compatible with the filtration $F_{i}$ in the sense that

$$
\left(F_{i} K_{l}^{\mathrm{sp}}(H)\right)\left(F_{j} K_{l}^{\mathrm{sp}}(H)\right) \subseteq F_{i+j} K_{l}^{\mathrm{sp}}(H) .
$$

Proof. The representation $V_{1}$ is the restriction of the tautological 2-dimensional representation $\tilde{V}_{1}$ of $\mathrm{SL}_{2}\left(\overline{\mathbb{F}}_{l}\right)$. Applying [AJL, Lemma 3.1, Proposition 3.3 (iii)] with $\lambda=l-2$, we know that $\tilde{V}_{1} \otimes \operatorname{Sym}^{l-1} \tilde{V}_{1}$ is indecomposable, and by AJL, Lemma 4.1 (a)], the restriction $\mathbf{W}$ of this representation to $\mathrm{SL}_{2}\left(\mathbb{F}_{l}\right)$ is the injective hull of an irreducible representation of $\mathrm{SL}_{2}\left(\mathbb{F}_{l}\right)$ and therefore indecomposable. (This fact can also be read off from Table 1 of the same paper.). This gives claim (1).

By [AJL, Lemma 2.5], for $1 \leq i \leq l-2$, we have

$$
V_{i} \otimes V_{1} \cong V_{i-1} \oplus V_{i+1} .
$$

By induction on $j$, this implies that for $i, j \geq 0$ and $i+j \leq l-1$, we have the Clebsch-Gordan formula

$$
V_{i} \otimes V_{j} \cong V_{i+j} \oplus V_{i+j-2} \oplus \cdots \oplus V_{|i-j|} .
$$

For $i+j=p$ and $0<i<j$, we claim that

$$
V_{i} \otimes V_{j}=\mathbf{W} \oplus \bigoplus_{k=2}^{i} V_{l-2 k}
$$


The statement is trivial for $i=1$, and for $i \geq 2$,

$$
\begin{aligned}
V_{i-2} \otimes V_{j} \oplus V_{i} \otimes V_{j} & \cong\left(V_{i-2} \oplus V_{i}\right) \otimes V_{j} \\
& \cong V_{1} \otimes\left(V_{i-1} \otimes V_{j}\right) \\
& \cong V_{1} \otimes\left(V_{l-1} \oplus V_{l-3} \oplus \cdots \oplus V_{l+1-2 i}\right) \\
& \cong \mathbf{W} \oplus\left(V_{l-2} \oplus V_{l-4}\right) \oplus \cdots \oplus\left(V_{l+2-2 i} \oplus V_{l-2 i}\right)
\end{aligned}
$$

As

$$
V_{i-2} \otimes V_{j}=V_{l-2} \oplus V_{l-4} \oplus \cdots \oplus V_{l+2-2 i},
$$

Krull-Schmidt implies our claim, which in turn implies (2).

Let

$$
\Lambda_{V_{1}}(t):=1-\left[V_{1}\right] t+t^{2} \in K_{l}^{\mathrm{sp}}(H)[t] .
$$

The analogy between the $(\bmod l)$ representation theory of $H$ and the (complex) representation theory of $\mathrm{SL}_{2}(\mathbb{C})$ might suggest the possibility that $\zeta_{V_{1}}(t)=\Lambda_{V_{1}}(t)^{-1}$, but this turns out not to be true. Instead (2.1) implies

$$
\Lambda_{V_{1}}(t) \zeta_{V_{1}}(t) \equiv 1+\left(\left[V_{l-2}\right]+\left[V_{l}\right]-[\mathbf{W}]\right) t^{l} \quad\left(\bmod t^{l+1}\right) .
$$

Note that since $\mathbf{W}$ is indecomposable, the $t^{l}$ coefficient of $\Lambda(t) \zeta_{V_{1}}(t)$ is nonzero. This phenomenon, as it arises in the case of the representation $V_{1} \otimes V_{1}$ of $\mathrm{SL}_{2}\left(\mathbb{F}_{l}\right) \times \mathrm{SL}_{2}\left(\mathbb{F}_{l}\right)$ is the key to our proof of irrationality.

Henceforth $G=\mathrm{SL}_{2}\left(\mathbb{F}_{l}\right) \times \mathrm{SL}_{2}\left(\mathbb{F}_{l}\right)$. For non-negative integers $n$, we define

$$
F_{n} K_{l}^{\mathrm{sp}}(G):=T\left(F_{n} K_{l}^{\mathrm{sp}}(H) \otimes F_{n} K_{l}^{\mathrm{sp}}(H)\right) .
$$

In particular, for $0 \leq n \leq l-1$,

$$
F_{n} K_{l}^{\mathrm{sp}}(G)=\operatorname{Span}_{\mathbb{Z}}\left\{\left[V_{i} \otimes V_{j}\right] \mid 0 \leq i, j \leq n\right\} .
$$

Proposition 2.3. For $0 \leq n \leq l-1$, we have

$$
\operatorname{Sym}^{n}\left(V_{1} \otimes V_{1}\right) \cong \sum_{i=0}^{\lfloor n / 2\rfloor} V_{n-2 i} \otimes V_{n-2 i} .
$$

Proof. First of all, the symmetric power is a quotient of

$$
\left(V_{1} \otimes V_{1}\right)^{\otimes n}=V_{1}^{\otimes n} \otimes V_{1}^{\otimes n},
$$

which by (2.1) and induction on $n$ is a direct sum of expressions of the form $V_{i} \otimes V_{j}$ with $i, j \leq n$. Thus $\operatorname{Sym}^{n}\left(V_{1} \otimes V_{1}\right)$ is itself a direct sum of such expressions. Writing

$$
\operatorname{Sym}^{n}\left(V_{1} \otimes V_{1}\right)=\bigoplus_{0 \leq i, j \leq n}\left(V_{i} \otimes V_{j}\right)^{a_{i, j}},
$$

it remains to prove that $a_{i, j}$ is 0 except when $i=j \in\{n, n-2, n-4, \ldots\}$, in which case it is 1 . 
Restricting to $H \times\{1\}$, we obtain the isomorphism of $H$-modules

$$
\bigoplus_{0 \leq i, j \leq n} V_{i}^{a_{i, j}(j+1)} \cong \operatorname{Sym}^{n}\left(V_{1} \oplus V_{1}\right) \cong \bigoplus_{a+b=n} V_{a} \otimes V_{b} \cong \bigoplus_{k=0}^{\lfloor n / 2\rfloor} V_{n-2 k}^{n-2 k+1},
$$

the last isomorphism following from (2.2). Thus, $a_{i, j}(j+1) \leq i+1$ for all $i, j \leq n$. By symmetry, also $a_{i, j}(i+1) \leq j+1$. Thus, $a_{i, j} \leq 1$ with equality only if $i=j$. Comparing with (2.4), we see that $a_{i, i}=1$ exactly for $i \in\{n, n-2, n-4, \ldots\}$.

Proposition 2.4. Define

$$
\Lambda_{V_{1} \otimes V_{1}}(t):=1-\left[V_{1} \otimes V_{1}\right] t+\left(\left[V_{2} \otimes V_{0}\right]+\left[V_{0} \otimes V_{2}\right]\right) t^{2}-\left[V_{1} \otimes V_{1}\right] t^{3}+t^{4} .
$$

Then

$$
\Lambda_{V_{1} \otimes V_{1}}(t) \zeta_{V_{1} \otimes V_{1}}(t) \equiv 1 \quad\left(\bmod t^{l}\right) .
$$

Proof. This is the special case $V:=V_{1} \otimes V_{1}$ of the general congruence formula

$$
\left(\sum_{i=0}^{\operatorname{dim} V}(-1)^{i}\left[\wedge^{i} V\right] t^{i}\right) \zeta_{V}(t) \equiv 1 \quad\left(\bmod t^{l}\right)
$$

Equivalently, we claim that for $1 \leq k<l$, we have

$$
\sum_{i+j=k}(-1)^{i}\left[\wedge^{i} V \otimes \operatorname{Sym}^{j} V\right]=0 .
$$

For every object $W$ of a $\lambda$-ring, we have the identity

$$
\sum_{i+j=k} \lambda^{i}(W) \lambda^{j}(-W)=0
$$

If $W$ is a finite-dimensional complex vector space regarded as an object of the representation ring of $\mathrm{GL}(W)$, it is easy to see by the splitting principle that $(-1)^{j} \lambda^{j}(-W)$ corresponds to $\operatorname{Sym}^{j} W$. If $\epsilon_{i, j}$ in the group ring $\mathbb{Z}[1 / k !]\left[S_{k}\right]$ denotes the projector which maps $W^{\otimes k}=W^{\otimes i+j}$ onto $\wedge^{i} W \otimes \operatorname{Sym}^{j} W$, this implies

$$
\sum_{i+j=k}(-1)^{i} \epsilon_{i, j}=(-1)^{i+j} \sum_{i+j=k}(-1)^{j} \epsilon_{i, j}=0 .
$$

As $k$ ! is invertible in $l$, this reduces to the same identity over $\mathbb{F}_{l}$, which implies the identity (2.6) for group representations in characteristic $l$.

We now come to the key lemma. Let $R$ be a ring containing $K_{l}^{\mathrm{sp}}(G)$. Let $A(t), B(t) \in R[t]$ denote polynomials with $A(0)=B(0)=1$, and let $\overline{\mathbb{F}}_{l}^{k}$ denote the trivial representation of $G$ of dimension $k$.

Lemma 2.5. If $A(t)$ and $B(t)$ are as above, $a_{i} \in R$ for all $i \geq 0$,

$$
A(t)=B(t)\left(a_{0}+a_{1} t+a_{2} t^{2}+\cdots\right),
$$

and

$$
a_{i}=\left[\operatorname{Sym}^{i}\left(\left(V_{1} \otimes V_{1}\right) \oplus \overline{\mathbb{F}}_{l}^{k}\right)\right] \in K_{l}^{\mathrm{sp}}(G)
$$


for $i \leq \operatorname{deg} A+\operatorname{deg} B+k+4<l$, then $a_{i} \in K_{l}^{\mathrm{sp}}(G)$ for all $i \geq 0$, and $a_{l}-[\mathbf{W} \otimes \mathbf{W}]+\left[\mathbf{W} \otimes V_{l-2}\right]+\left[V_{l-2} \otimes \mathbf{W}\right] \in F_{l-1} K_{l}^{\mathrm{sp}}(G)$.

Proof. For any $G$-representation $V$,

$$
\operatorname{Sym}^{n}\left(V \oplus \overline{\mathbb{F}}_{l}\right) \cong \bigoplus_{i=0}^{n} \operatorname{Sym}^{i} V .
$$

Thus,

$$
(1-t) \sum_{i=0}^{n}\left[\operatorname{Sym}^{i}\left(V \oplus \overline{\mathbb{F}}_{l}\right)\right] t^{i} \equiv \sum_{i=0}^{n}\left[\operatorname{Sym}^{i} V\right] t^{i} \quad\left(\bmod t^{n-1}\right) .
$$

Iterating,

$$
\begin{aligned}
(1-t)^{k} \sum_{i=0}^{\infty} a_{i} t^{i} & \equiv(1-t)^{k} \sum_{i=0}^{n}\left[\operatorname{Sym}^{i}\left(\left(V_{1} \otimes V_{1}\right) \oplus \overline{\mathbb{F}}_{l}^{k}\right)\right] t^{i} \\
& \equiv \sum_{i=0}^{n}\left[\operatorname{Sym}^{i}\left(V_{1} \otimes V_{1}\right)\right] t^{i} \quad\left(\bmod t^{n+1}\right)
\end{aligned}
$$

Thus, replacing $B(t)$ with $B(t)(1-t)^{k}$, we may assume $k=0$, which means $a_{i}=\left[\operatorname{Sym}^{i}\left(V_{1} \otimes V_{1}\right)\right]$ for $0 \leq i \leq \operatorname{deg} A+\operatorname{deg} B+4$.

Defining $\Lambda_{V_{1} \otimes V_{1}}(t)$ as in (2.5) and multiplying (2.7) by $B(t) \Lambda_{V_{1} \otimes V_{1}}(t)$, we get

$$
\begin{aligned}
A(t) \Lambda_{V_{1} \otimes V_{1}}(t) \equiv B(t) \Lambda_{V_{1} \otimes V_{1}}(t) \sum_{i=0}^{\infty} a_{i} t^{i} & \equiv B(t) \Lambda_{V_{1} \otimes V_{1}}(t) \sum_{i=0}^{\infty}\left[\operatorname{Sym}^{i}\left(V_{1} \otimes V_{1}\right)\right] t^{i} \\
& \equiv B(t) \quad\left(\bmod t^{\operatorname{deg} A+\operatorname{deg} B+5}\right) .
\end{aligned}
$$

As $\operatorname{deg}\left(A(t) \Lambda_{V_{1} \otimes V_{1}}(t)-B(t)\right)<\operatorname{deg} A+\operatorname{deg} B+5$, we have $A(t) \Lambda_{V_{1} \otimes V_{1}}(t)=$ $B(t)$, so

$$
A(t)\left(1-\Lambda_{V_{1} \otimes V_{1}}(t) \sum_{i=0}^{\infty} a_{i} t^{i}\right)=0 .
$$

As $A(t)$ is invertible in $R[[t]]$, this implies

$$
\Lambda_{V_{1} \otimes V_{1}}(t) \sum_{i=0}^{\infty} a_{i} t^{i}=1
$$

This gives a linear recurrence for the $a_{i}$ with coefficients in $K_{l}^{\mathrm{sp}}(G)$, and it follows that $a_{i} \in K_{l}^{\mathrm{sp}}(G)$ for all $i \geq 0$.

By Proposition 2.4.

$$
\zeta_{V_{1} \otimes V_{1}}(t) \equiv \sum_{i=0}^{\infty} a_{i} t^{i} \quad\left(\bmod t^{l}\right)
$$

This implies (2.8) for all $n \leq l-1$. 
Finally, matching $t^{l}$ coefficients in (2.9), we get

$$
a_{l}=a_{1} a_{l-1}-\left(\left[V_{2} \otimes V_{0}\right]+\left[V_{0} \otimes V_{2}\right]\right) a_{l-2}+a_{1} a_{l-3}-a_{l-4} .
$$

Modulo classes in $F_{l-1} K_{l}^{\mathrm{sp}}(G)$, the right hand side reads

$$
\left[V_{1} \otimes V_{1}\right]\left[V_{l-1} \otimes V_{l-1}\right]-\left(\left[V_{2} \otimes V_{0}\right]+\left[V_{0} \otimes V_{2}\right]\right)\left[V_{l-2} \otimes V_{l-2}\right],
$$

which, by (2.2), further reduces modulo $\operatorname{Span}_{\mathbb{Z}}\left\{\left[V_{i} \otimes V_{j}\right] \mid 0 \leq i, j \leq l-1\right\}$ to

$$
[\mathbf{W} \otimes \mathbf{W}]-\left[\mathbf{W} \otimes V_{l-2}\right]-\left[V_{l-2} \otimes \mathbf{W}\right]
$$

Lemma 2.6. Suppose the hypotheses of Lemma2.5 are satisfied. If elements $b_{i} \in R$ satisfy

$$
\sum_{i=0}^{\infty} b_{i} t^{i}=\prod_{r=1}^{\infty} \sum_{j=0}^{\infty} a_{j} t^{j r}
$$

then $b_{i} \in K_{l}^{\mathrm{sp}}(G)$ for all $i \geq 0$, and

$$
b_{l} \equiv[\mathbf{W} \otimes \mathbf{W}]-\left[\mathbf{W} \otimes V_{l-2}\right]-\left[V_{l-2} \otimes \mathbf{W}\right] \quad\left(\bmod F_{l-1} K_{l}^{\mathrm{sp}}(G)\right) .
$$

Proof. Since $a_{i} \in K_{l}^{\mathrm{sp}}(G)$ for all $i$ and $a_{i} \in F_{i} K_{l}^{\mathrm{sp}}(G)$ for $0 \leq i \leq l-1$, (2.10) implies by induction on $i$ that $b_{i} \in K_{l}^{\mathrm{sp}}(G)$ for all $i \geq 0$ and $b_{i} \in F_{i} K_{l}^{\mathrm{sp}}(G)$ for $0 \leq i \leq l-1$. As $b_{l}-a_{l}$ is a linear combination of products $a_{i_{1}} \cdots a_{i_{k}}$ with $i_{1}+\cdots+i_{n} \leq l-1$, the claim for $b_{l}$ follows.

We note for future reference that the relationship (2.10) between the $a_{i}$ and the $b_{i}$ is significant because it is the relationship between the cohomology of the $i$ th symmetric power of $X$ and the $i$ th Hilbert scheme of $X$.

\section{A FAMily OF MOtiviC MEASURES}

In this section, we construct the motivic measures needed for the proof of our main theorem.

Let $k$ be a subfield of $\mathbb{C}$. Let $\bar{k}$ denote the algebraic closure of $k$ in $\mathbb{C}$, and set $G_{k}:=\operatorname{Gal}(\bar{k} / k)$. We define $\bar{X}:=X \times_{\operatorname{Spec} k}$ Spec $\bar{k}$ for any variety $X / k$. We regard the étale cohomology groups $H^{i}\left(\bar{X}, \overline{\mathbb{F}}_{l}\right)$ and $H_{c}^{i}\left(\bar{X}, \overline{\mathbb{F}}_{l}\right)$ as $G_{k}$-representations. They are obtained by extension of scalars from the $G_{k}$-representations $H^{i}\left(\bar{X}, \mathbb{F}_{l}\right)$ and $H_{c}^{i}\left(\bar{X}, \mathbb{F}_{l}\right)$ respectively.

Our construction depends on the Bittner construction [Bi]. In order to carry it out, we need the following theorem:

Theorem 3.1. Let $X$ be a non-singular projective variety over $k$ and $Y \subset X$ a nonsingular closed subvariety of codimension $r, X^{\prime}$ the blow up of $X$ along $Y$, and $Y^{\prime}$ the inverse image of $Y$ in $X^{\prime}$. Then for any $q$ there is a natural direct sum decomposition of $G_{k}$-modules

$$
H^{q}\left(\bar{X}^{\prime}, \overline{\mathbb{F}}_{l}\right)=H^{q}\left(\bar{X}, \overline{\mathbb{F}}_{l}\right) \oplus \bigoplus_{j=1}^{r-1} H^{q-2 j}\left(\bar{Y}, \overline{\mathbb{F}}_{l}(-j)\right)
$$


The analogue Theorem 3.1 is proved for $\mathbb{Z}_{l}$-coefficients (instead of $\overline{\mathbb{F}}_{l}$ ) in SGA 7, XVIII 2.2.2]. We essentially reproduce the argument (filling in some details) to prove it in our setting. We make use of the following proposition Mi, VI, 10.1]:

Proposition 3.2. Let $\bar{Y}$ be a smooth projective variety over $\bar{k}$ and let $\mathcal{E}$ be a vector bundle of rank $r$ over $\bar{Y}$. Let $\mathbb{P}(\mathcal{E}) \rightarrow \bar{Y}$ be the corresponding projective bundle. Then for each $q$ there is a natural isomorphism of $G_{k}$ modules

$$
H^{q}\left(\mathbb{P}(\mathcal{E}), \overline{\mathbb{F}}_{l}\right)=\bigoplus_{j \geq 0} H^{q-2 j}\left(\bar{Y}, \overline{\mathbb{F}}_{l}(-j)\right),
$$

where the summand for $j=0$ is the image of the map $p^{*}: H^{q}\left(\bar{Y}, \overline{\mathbb{F}}_{l}\right) \rightarrow$ $H^{q}\left(\mathbb{P}(\mathcal{E}), \overline{\mathbb{F}}_{l}\right)$.

Proof of Theorem 3.1. Consider the commutative diagram

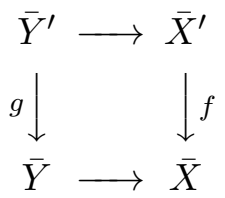

Note that the map $g: \bar{Y}^{\prime} \rightarrow \bar{Y}$ is the projective bundle corresponding to the normal vector bundle $N_{\bar{Y} / \bar{X}}$ of rank $r$ on $\bar{Y}$. Writing $\bar{U}:=\bar{X} \backslash \bar{Y} \cong$ $\bar{X}^{\prime} \backslash \bar{Y}^{\prime}=: \bar{U}^{\prime}$, we have the induced morphism of long exact sequences of cohomology with compact supports

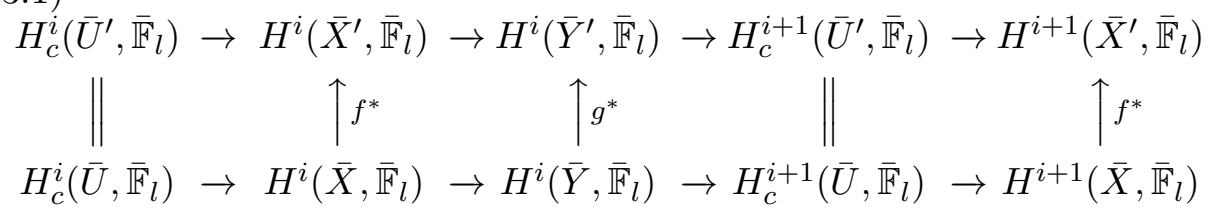

(where we use the fact that $H_{c}^{i}(-)$ coincides with $H^{i}(-)$ for the projective varieties $\left.\bar{X}, \bar{X}^{\prime}, \bar{Y}, \bar{Y}^{\prime}\right)$.

We know that the map $g^{*}$ is injective by Proposition 3.2. We claim that the arrows $f^{*}$ are also injective.

Lemma 3.3. For each $i$ the map $f^{*}: H^{i}\left(\bar{X}, \overline{\mathbb{F}}_{l}\right) \rightarrow H^{i}\left(\bar{X}^{\prime}, \overline{\mathbb{F}}_{l}\right)$ is injective. Moreover it has a natural left inverse.

Proof. We may assume that $\bar{X}$ and hence also $\bar{X}^{\prime}$ is connected, so that $H^{0}\left(\bar{X}, \overline{\mathbb{F}}_{l}\right)=H^{0}\left(\bar{X}^{\prime}, \overline{\mathbb{F}}_{l}\right)=\overline{\mathbb{F}}_{l}$. We denote by $1_{\bar{X}} \in H^{0}\left(\bar{X}, \overline{\mathbb{F}}_{l}\right)$ and $1_{\bar{X}^{\prime}} \in$ $H^{0}\left(\bar{X}^{\prime}, \overline{\mathbb{F}}_{l}\right)$ the corresponding generators. Clearly $f^{*}\left(1_{\bar{X}}\right)=1_{\bar{X}^{\prime}}$.

Let $n=\operatorname{dim} \bar{X}=\operatorname{dim} \bar{X}^{\prime}$. Recall that Poincaré duality Mi, VI, Theorem 11.1] gives a canonical nondegenerate Galois-equivariant pairing

$$
H^{r}\left(\bar{X}, \overline{\mathbb{F}}_{l}\right) \times H^{2 n-r}\left(\bar{X}, \overline{\mathbb{F}}_{l}(n)\right) \stackrel{\cup}{\longrightarrow} H^{2 n}\left(\bar{X}, \overline{\mathbb{F}}_{l}(n)\right) \stackrel{\eta_{\bar{X}}}{\longrightarrow} \overline{\mathbb{F}}_{l}
$$

where the map on the left is cup-product and $\eta_{\bar{X}}: H^{2 n}\left(\bar{X}, \overline{\mathbb{F}}_{l}(n)\right) \rightarrow \overline{\mathbb{F}}_{l}$ is the trace map isomorphism. It has the property that for every closed point 
$P$ we have $\eta_{\bar{X}}\left(\operatorname{cl}_{X}(P)\right)=1 \in \overline{\mathbb{F}}_{l}$, where $\operatorname{cl}_{\bar{X}}(P) \in H^{2 n}\left(\bar{X}, \overline{\mathbb{F}}_{l}(n)\right)$ is the image under the Gysin map $H^{0}\left(P, \overline{\mathbb{F}}_{l}\right) \rightarrow H^{2 n}\left(\bar{X}, \overline{\mathbb{F}}_{l}(n)\right)$ of $1_{P}$ [Mi, VI, p.269]. The same applies to $\bar{X}^{\prime}$.

Choose a closed point $P \in \bar{X} \backslash \bar{Y}$. Then $f^{-1}(P)=Q$ is a single point in $\bar{X}^{\prime}$. Hence it follows from [Mi, VI, Proposition 9.2] that the map $f^{*}$ : $H^{2 n}\left(\bar{X}, \overline{\mathbb{F}}_{l}(n)\right) \rightarrow H^{2 n}\left(\bar{X}^{\prime}, \overline{\mathbb{F}}_{l}(n)\right)$ takes $\mathrm{cl}_{\bar{X}}(P)$ to $\mathrm{cl}_{\bar{X}^{\prime}}(Q)$. It follows that the diagram

commutes.

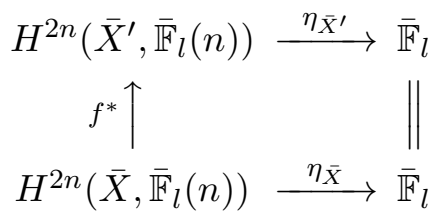

Poincaré dualities for $H^{\bullet}(\bar{X})$ and for $H^{\bullet}\left(\bar{X}^{\prime}\right)$ induce the pushforward map

$$
f_{*}: H^{i}\left(\bar{X}^{\prime}, \overline{\mathbb{F}}_{l}\right) \rightarrow H^{i}\left(\bar{X}, \overline{\mathbb{F}}_{l}\right)
$$

such that $\mathrm{f}_{*}\left(x^{\prime} \cup f^{*}(x)\right)=f_{*}\left(x^{\prime}\right) \cup x$ for $x \in H^{\bullet}(\bar{X})$ and $x^{\prime} \in H^{\bullet}\left(\bar{X}^{\prime}\right)$ Mi, VI, Remark 11.6]. We have $f_{*}\left(1_{\bar{X}^{\prime}}\right)=d \cdot 1_{\bar{X}}$ for some $d \in \overline{\mathbb{F}}_{l}$. We claim that in fact $d=1$. Indeed, in the above notation we have

$$
\begin{aligned}
1 & =\eta_{\bar{X}^{\prime}}\left(\operatorname{cl}_{\bar{X}^{\prime}}(Q)\right) \\
& =\eta_{\bar{X}^{\prime}}\left(1_{\bar{X}^{\prime}} \cup \mathrm{cl}_{\bar{X}^{\prime}}(Q)\right) \\
& =\eta_{\bar{X}^{\prime}}\left(1_{\bar{X}^{\prime}} \cup f^{*}\left(\mathrm{cl}_{\bar{X}}(P)\right)\right) \\
& =\eta_{\bar{X}^{\prime}}\left(f_{*}\left(1_{\bar{X}^{\prime}}\right) \cup \mathrm{cl}_{\bar{X}}(P)\right) \\
& =d \cdot \eta_{\bar{X}}\left(1_{\bar{X}} \cup \mathrm{cl}_{\bar{X}}(P)\right) \\
& =d \cdot \eta_{\bar{X}}\left(\mathrm{cl}_{\bar{X}}(P)\right) \\
& =d
\end{aligned}
$$

It follows that $f_{*} f^{*}(x)=x$ for every $x \in H^{\bullet}\left(X, \overline{\mathbb{F}}_{l}\right)$. Indeed,

$$
f_{*} f^{*}(x)=f_{*}\left(1_{X^{\prime}} \cup f^{*}(x)\right)=f_{*}\left(1_{X^{\prime}}\right) \cup x=1_{X} \cup x=x
$$

This proves the lemma.

It follows from the injectivity of $f^{*}$ that the diagram (3.1) induces an isomorphism

$$
\text { coker } f^{*} \stackrel{\sim}{\rightarrow} \text { coker } g^{*} \text {. }
$$

The fact that $f^{*}$ has a canonical left-inverse allows us to identify $H^{i}\left(\bar{X}^{\prime}, \overline{\mathbb{F}}_{l}\right)$ with $H^{i}\left(\bar{X}, \overline{\mathbb{F}}_{l}\right) \oplus$ coker $f^{*}$ as $G_{k}$-modules. Thus

$$
H^{q}\left(\bar{X}^{\prime}, \overline{\mathbb{F}}_{l}\right) \stackrel{\sim}{\rightarrow} H^{q}\left(\bar{X}, \overline{\mathbb{F}}_{l}\right) \oplus \operatorname{coker} g^{*} \stackrel{\sim}{\rightarrow} H^{q}\left(\bar{X}, \overline{\mathbb{F}}_{l}\right) \oplus \bigoplus_{j=1}^{r-1} H^{q-2 j}\left(\bar{Y}, \overline{\mathbb{F}}_{l}(-j)\right)
$$

by Proposition 3.2 ,

Theorem 3.4. For each prime $l$ and every field $k$ of characteristic 0 , there exists a unique motivic measure $\mu_{l}: K_{0}\left(\operatorname{Var}_{k}\right) \rightarrow K_{l}^{\mathrm{sp}}\left(G_{k}\right)$ satisfying

$$
\mu_{l}([X])=\sum_{i=0}^{2 \operatorname{dim} X}\left[H^{i}\left(\bar{X}, \overline{\mathbb{F}}_{l}\right)\right]
$$


for all projective non-singular varieties $X$.

Proof. By Bittner's theorem [Bi], it suffices to prove that $\mu_{l}([X \times Y])=$ $\mu_{l}([X]) \mu_{l}([Y])$ whenever $X$ and $Y$ are non-singular projective varieties and that whenever $X$ is a non-singular projective variety, $Y$ a non-singular closed subvariety, $X^{\prime}$ the blow up of $X$ along $Y$ and $Y^{\prime}$ the inverse image of $Y$ in $X^{\prime}$ then

$$
\mu_{l}\left(\left[X^{\prime}\right]\right)-\mu_{l}([X])=\mu_{l}\left(\left[Y^{\prime}\right]\right)-\mu_{l}([Y]) .
$$

The first property follows immediately from the Künneth formula [Mi, VI, 8.13]. The second follows from Theorem 3.1 and Proposition 3.2 .

Definition 3.5. We define the motivic measure $\nu_{l}: K_{0}\left(\operatorname{Var}_{k}\right) \rightarrow K_{l}^{\mathrm{sp}}\left(G_{k\left(\zeta_{l}\right)}\right)$ to be the composition of $\mu_{l}$ with the restriction map $K_{l}^{\mathrm{sp}}\left(G_{k}\right) \rightarrow K_{l}^{\mathrm{sp}}\left(G_{k\left(\zeta_{l}\right)}\right)$.

In the application to the main theorem, we will always take $k=\mathbb{Q}$.

\section{Galois Representations}

Proposition 4.1. There exist elliptic curves $E_{1}$ and $E_{2}$ over $\mathbb{Q}$ such that for all sufficiently large primes $l$, there exist linearly disjoint Galois extensions $K_{1}$ and $K_{2}$ of $\mathbb{Q}\left(\zeta_{l}\right)$ such that the $(\bmod l)$ Galois representations of $G_{\mathbb{Q}\left(\zeta_{l}\right)}$ acting on $H^{1}\left(\bar{E}_{i}, \mathbb{F}_{l}\right)$ have kernels $G_{K_{i}}$ and images isomorphic to $\mathrm{SL}_{2}\left(\mathbb{F}_{l}\right)$.

Proof. Fix primes $q, r \geq 5$. Let $E_{1}$ and $E_{2}$ be any elliptic curves over $\mathbb{Q}$ with multiplicative reduction at $q$ and such that $E_{1}$ and $E_{2}$ have respectively good ordinary reduction and good supersingular reduction at $r$. (For instance, if $q=11$ and $r=5$, the curves given in Cremona notation by $E_{1}:=33 \mathrm{a} 1$ and $E_{2}:=11$ a1 satisfy these conditions.) Let $\rho_{i}^{l}$ denote the homomorphism from the absolute Galois group $G_{\mathbb{Q}}$ to $\mathrm{GL}\left(H^{1}\left(\bar{E}_{i}, \mathbb{F}_{l}\right)\right) \cong \mathrm{GL}_{2}\left(\mathbb{F}_{l}\right)$.

Neither $E_{1}$ nor $E_{2}$ can have complex multiplication, since every CM curve has integral $j$-invariant [Si, II Theorem 6.1], while an elliptic curve with multiplicative reduction at $q$ cannot have $q$-adically integral $j$-invariant $\underline{\text { Si }}$, Table 4.1]. By Serre's theorem [Se, for $l$ sufficiently large, the $\rho_{i}^{l}$ is surjective, so the image of $G_{\mathbb{Q}\left(\zeta_{l}\right)}$ in $\operatorname{GL}\left(H^{1}\left(\bar{E}_{i}, \mathbb{F}_{l}\right)\right)$ is $\mathrm{SL}_{2}\left(\mathbb{F}_{l}\right)$. We assume this holds and that $l \geq 5$. Let $\bar{\rho}_{i}^{l}: G_{\mathbb{Q}} \rightarrow \mathrm{PGL}_{2}\left(\mathbb{F}_{l}\right)$ denote the composition of $\rho_{i}^{l}$ with the quotient map $\mathrm{GL}_{2}\left(\mathbb{F}_{l}\right) \rightarrow \mathrm{PGL}_{2}\left(\mathbb{F}_{l}\right)$.

Suppose that $\left.\rho_{1}^{l}\right|_{G_{\mathbb{Q}\left(\zeta_{l}\right)}}=\left.\rho_{2}^{l}\right|_{G_{\mathbb{Q}\left(\zeta_{l}\right)}}$. As the common image of the two representations has trivial centralizer in $\mathrm{PGL}_{2}\left(\mathbb{F}_{l}\right)$, it follows that $\bar{\rho}_{1}^{l}=\bar{\rho}_{2}^{l}$. Thus, $\rho_{1}^{l}=\rho_{2}^{l} \otimes \chi$ for some character $\chi$ of $\operatorname{Gal}\left(\mathbb{Q}\left(\zeta_{l}\right) / \mathbb{Q}\right)$. Taking determinant of both sides, $\chi^{2}=1$.

The representations $\rho_{i}^{l}$ are both unramified at $r$, so $\operatorname{Tr}\left(\rho_{i}^{l}\left(\operatorname{Frob}_{r}\right)\right)$ is well defined, and the two traces are related by a factor of $\chi\left(\right.$ Frob $\left._{r}\right)= \pm 1$. This is impossible since the trace of Frob ${ }_{r}$ is zero for $E_{2}$ but not for $E_{1}$.

Now $\rho_{1}^{l}$ and $\rho_{2}^{l}$ together give an injective homomorphism $\rho_{12}^{l}$

$$
\operatorname{Gal}\left(K_{1} K_{2} / \mathbb{Q}\left(\zeta_{l}\right)\right) \rightarrow \mathrm{SL}_{2}\left(\mathbb{F}_{l}\right) \times \mathrm{SL}_{2}\left(\mathbb{F}_{l}\right)
$$


whose image projects onto $\mathrm{SL}_{2}\left(\mathbb{F}_{l}\right)$ on both factors. As the only normal subgroups of $\mathrm{SL}_{2}\left(\mathbb{F}_{l}\right)$ are the group itself, $\{ \pm 1\}$ and $\{1\}$, applying Goursat's lemma to the image of $\rho_{12}^{l}$, either this image is all of $\mathrm{SL}_{2}\left(\mathbb{F}_{l}\right) \times \mathrm{SL}_{2}\left(\mathbb{F}_{l}\right)$, in which case $\rho_{12}^{l}$ is an isomorphism, or $\bar{\rho}_{1}$ and $\bar{\rho}_{2}$ coincide on $\operatorname{Gal}\left(K_{1} K_{2} / \mathbb{Q}\left(\zeta_{l}\right)\right)$. We have seen that the latter is impossible, so the proposition follows.

We remark that assuming the Frey-Mazur conjecture is true, Proposition 4.1 is true for any two non-CM elliptic curves which are not isogenous over $\overline{\mathbb{Q}}$.

If $l$ is a prime, $\Sigma$ is a group of order prime to $l$, and $V$ a finite dimensional $\Sigma$-representation over $\overline{\mathbb{F}}_{l}$, then the map

$$
v \mapsto|\Sigma|^{-1} \sum_{\sigma \in \Sigma} \sigma v
$$

induces a natural isomorphism $V_{\Sigma} \rightarrow V^{\Sigma}$, from coinvariants to invariants. In what follows, we do not distinguish between these spaces; in particular, we identify the symmetric $n$th power with the symmetric tensors when $n<l$.

Proposition 4.2. Let $X$ be a variety over a field $k \subseteq \mathbb{C}, l$ a prime, and $\Sigma$ a finite group whose order is prime to $l$. For any $\Sigma$-action on $X$ defined over $k$ and for every $q \geq 0$, there is a canonical isomorphism of $G_{k}$-modules

$$
H^{q}\left(\overline{X / \Sigma}, \overline{\mathbb{F}}_{l}\right) \stackrel{\sim}{\rightarrow} H^{q}\left(\bar{X}, \overline{\mathbb{F}}_{l}\right)^{\Sigma} .
$$

Proof. As the morphism $X \rightarrow X / \Sigma$ is defined over $k$, the natural map $H^{q}\left(\overline{X / \Sigma}, \overline{\mathbb{F}}_{l}\right) \rightarrow H^{q}\left(\bar{X}, \overline{\mathbb{F}}_{l}\right)$ respects $G_{k^{-}}$-actions, and its image of course lies in the space of $\Sigma$-invariants. It remains to see that this is an isomorphism. As $k \subseteq \mathbb{C}$, we can use the comparison theorem with the ordinary cohomology of $X(\mathbb{C})$. The statement for cohomology of CW complexes is due to Grothendieck [Gr, 5.2.3].

Corollary 4.3. Let $X$ be a variety over a field $k \subseteq \mathbb{C}, l$ a prime, and $n<l$ is a non-negative integer, then

$$
H^{\bullet}\left(\overline{\operatorname{Sym}^{n} X}, \overline{\mathbb{F}}_{l}\right) \stackrel{\sim}{\rightarrow} \operatorname{Sym}^{n} H^{\bullet}\left(\bar{X}, \overline{\mathbb{F}}_{l}\right),
$$

where $\operatorname{Sym}^{n}$ is taken in the sense of $\mathbb{Z} / 2 \mathbb{Z}$-graded $G_{k}$-representations (i.e., if $V=V^{0} \oplus V^{1}, \operatorname{Sym}^{n} V$ means $\bigoplus_{i+j=n} \operatorname{Sym}^{i} V^{0} \otimes \wedge^{j} V^{1}$.)

Proof. By the Künneth formula,

$$
H^{\bullet}\left(\bar{X}^{n}, \overline{\mathbb{F}}_{l}\right) \stackrel{\sim}{\rightarrow} H^{\bullet}\left(\bar{X}, \overline{\mathbb{F}}_{l}\right)^{\otimes n} .
$$

The corollary follows by applying Proposition 4.2 to $\Sigma:=\Sigma_{n}$.

Note that if $H^{\bullet}\left(\bar{X}, \overline{\mathbb{F}}_{l}\right)$ is zero in odd degrees, then the action of $\Sigma_{n}$ is the usual permutation action on tensor factors, and the symmetric $n$th power can therefore be taken in the usual sense of $G_{k}$-representations. There is no distinction between the alternating sum of cohomology and the total cohomology so we can work with Galois representations rather than virtual representations. 
Theorem 4.4. Let $E_{1}, E_{2}, K_{1}, K_{2}$ be as in Proposition 4.1. Let $X^{\prime}$ denote the K3 surface obtained by blowing up the nodes of the Kummer surface

$$
X:=\left(E_{1} \times E_{2}\right) /\langle\iota\rangle,
$$

where $\iota$ is multiplication by -1 . For $l$ sufficiently large, there is an isomorphism

$$
\operatorname{Gal}\left(K_{1} K_{2} / \mathbb{Q}\left(\zeta_{l}\right)\right) \rightarrow \mathrm{SL}_{2}\left(\mathbb{F}_{l}\right) \times \mathrm{SL}_{2}\left(\mathbb{F}_{l}\right),
$$

and with respect to this isomorphism,

$$
\nu_{l}\left(\left[X^{\prime}\right]\right)=\operatorname{Res}_{G_{\mathbb{Q}\left(\zeta_{l}\right)}}^{\mathrm{SL}_{2}\left(\mathbb{F}_{l}\right)^{2}}\left[\overline{\mathbb{F}}_{l}^{20} \oplus V_{1} \otimes V_{1}\right] .
$$

Proof. The action of $\iota$ on the $l$-torsion of $E_{1} \times E_{2}$ and therefore on

$$
H^{1}\left(\overline{E_{1} \times E_{2}}, \overline{\mathbb{F}}_{l}\right) \stackrel{\sim}{\rightarrow} \operatorname{Hom}\left(\bar{E}_{1}[l] \times \bar{E}_{2}[l], \overline{\mathbb{F}}_{l}\right)
$$

is by multiplication by -1 ; as the cohomology of every abelian variety is generated by $H^{1}, \iota$ acts on $H^{q}\left(\overline{E_{1} \times E_{2}}, \overline{\mathbb{F}}_{l}\right)$ by multiplication by $(-1)^{q}$. Assuming $l>2$, It follows from Proposition 4.2 that $H^{q}\left(\bar{X}, \overline{\mathbb{F}}_{l}\right)$ is 0 for $q$ odd and is

$$
H^{2}\left(\overline{E_{1} \times E_{2}}, \overline{\mathbb{F}}_{l}\right) \cong \overline{\mathbb{F}}_{l}(1) \oplus H^{1}\left(\bar{E}_{1}, \overline{\mathbb{F}}_{l}\right) \otimes H^{1}\left(\bar{E}_{2}, \overline{\mathbb{F}}_{l}\right) \oplus \overline{\mathbb{F}}_{l}(1)
$$

for $q=2$. For $q=0$ and $q=4$, we get $\overline{\mathbb{F}}_{l}$ and $\overline{\mathbb{F}}_{l}(2)$ respectively.

Let $Y$ denote the set of 16 double points on $X$ and $Y^{\prime}$ the inverse image of $Y$ in $X^{\prime}$, consisting of 16 copies of $\mathbb{P}^{1}$. Let $U:=X \backslash Y \cong X^{\prime} \backslash Y^{\prime}$. The excision sequence for $U \subset X$ gives $H_{c}^{i}\left(\bar{U}, \overline{\mathbb{F}}_{l}\right) \stackrel{\sim}{\rightarrow} H^{i}\left(\bar{X}, \overline{\mathbb{F}}_{l}\right)$ for $i \geq 2$, and if $l$ is sufficiently large, the excision sequence for $U \subset X^{\prime}$ gives a short exact sequence of $G_{\mathbb{Q}\left(\zeta_{l}\right)}$-modules

$$
0 \rightarrow H^{2}\left(\bar{X}, \overline{\mathbb{F}}_{l}\right) \rightarrow H^{2}\left(\bar{X}^{\prime}, \overline{\mathbb{F}}_{l}\right) \rightarrow \overline{\mathbb{F}}_{l}(1)^{16} \rightarrow 0
$$

and therefore

$$
0 \rightarrow H^{1}\left(\bar{E}_{1}, \overline{\mathbb{F}}_{l}\right) \otimes H^{1}\left(\bar{E}_{2}, \overline{\mathbb{F}}_{l}\right) \rightarrow H^{2}\left(\bar{X}^{\prime}, \overline{\mathbb{F}}_{l}\right) \rightarrow \overline{\mathbb{F}}_{l}(1)^{18} \rightarrow 0 .
$$

Regarding $H^{2}\left(\bar{X}^{\prime}, \overline{\mathbb{F}}_{l}\right)$ as a representation of $G_{\mathbb{Q}\left(\zeta_{l}\right)}$, it factors through the Galois group $\operatorname{Gal}\left(K_{1} K_{2} / \mathbb{Q}\left(\zeta_{l}\right)\right)$ which is isomorphic to $\mathrm{SL}_{2}\left(\mathbb{F}_{l}\right)^{2}$. As $\mathrm{SL}_{2}\left(\mathbb{F}_{l}\right)^{2}$ representation it is an extension of an 18-dimensional trivial representation by $V_{1} \otimes V_{1}$. If $l$ is sufficiently large, this extension is trivial, since all indecomposable $\overline{\mathbb{F}}_{l}$-representations of $\mathrm{SL}_{2}\left(\mathbb{F}_{l}\right)$ which are not irreducible have dimension at least $l-2$ [AJL, Corollary 4.3]. As $H^{0}\left(\bar{X}^{\prime}, \overline{\mathbb{F}}_{l}\right)$ and $H^{4}\left(\bar{X}^{\prime}, \overline{\mathbb{F}}_{l}\right)$ are trivial one-dimensional representations of $G_{\mathbb{Q}\left(\zeta_{l}\right)}$ and $H^{1}\left(\bar{X}^{\prime}, \overline{\mathbb{F}}_{l}\right)=H^{3}\left(\bar{X}^{\prime}, \overline{\mathbb{F}}_{l}\right)=$ 0 , the theorem follows. 


\section{Hilbert SChemes of SURFACES}

This section is devoted to a proof of an identity relating the classes of the Hilbert schemes of a non-singular surface to those of symmetric powers of the surface.

Theorem 5.1. If $X$ is a non-singular surface over a field $k$, we have an identity of power series in $K_{0}\left(\operatorname{Var}_{k}\right)$ as follows:

$$
\sum_{n=0}^{\infty}\left[X^{[n]}\right] t^{n}=\prod_{i=1}^{\infty} \zeta_{X}\left(E^{i-1} t^{i}\right) .
$$

This theorem is due to Göttsche $\mathrm{Go}$ in the case that $k$ is algebraically closed and of characteristic zero. His proof goes through in essentially the same way for any field. The only point requiring elaboration is the key identity (due to Ellingsrud and Strømme [ES, Theorem 1.1(iv)] in the case $k=\mathbb{C}$ ) for the class in $K_{0}\left(\operatorname{Var}_{k}\right)$ of $\left[R_{n}\right]$. Here $R_{n}$ denotes the (reduced) Hilbert scheme of codimension $n$ ideals of $k[[x, y]]$, or, equivalently, in $k[[x, y]] /(x, y)^{n}$, or, yet again, if $R$ is any $k$-algebra of Krull dimension 2 and $\mathfrak{m}$ any maximal ideal of $R$ such that $\operatorname{dim}_{k} R / \mathfrak{m}=1$ and $R$ is regular at $\mathfrak{m}$, the Hilbert scheme of $\mathfrak{m}$-primary codimension $n$ ideals of $R$. It is convenient to take $R=k[x, y]$ and $\mathfrak{m}=(x, y)$.

The following identity holds for general $k$.

Proposition 5.2. Let $k$ be any field, and let $n$ be a positive integer. Then

$$
\left[R_{n}\right]=\sum_{\beta \in P(n)}\left[\mathbb{A}^{n-|\beta|}\right]
$$

where $P(n)$ denotes the set of partitions $\beta$ of $n$, and $|\beta|$ is the number of parts of a partition.

We prove the proposition by giving an explicit "cell decomposition" of $R_{n}$ and explicit parametrizations of the cells. Toward this end, we introduce the following notation. Let $\beta$ and $\lambda$ be mutually dual partitions (i.e., partitions whose Ferrers diagrams are transpose to one another) with

$$
\begin{aligned}
& r=\beta_{1} \geq \beta_{2} \geq \cdots \geq \beta_{s}>\beta_{s+1}=0 \\
& s=\lambda_{1} \geq \lambda_{2} \geq \cdots \geq \lambda_{r}>\lambda_{r+1}=0 .
\end{aligned}
$$

Thus,

$$
\beta_{\lambda_{i}+1}<i \leq \beta_{\lambda_{i}}
$$

for $1 \leq i \leq r$, and

$$
\lambda_{\beta_{j}+1}<j \leq \lambda_{\beta_{j}}
$$

for $1 \leq j \leq s$. For $\beta$ (and therefore $\lambda$ ) fixed, we define the polynomial $\operatorname{ring} \mathcal{A}_{\beta}:=\mathbb{Z}\left[t_{i j}\right]$ where $1 \leq i<r$ and $1 \leq j \leq \lambda_{i+1}$ and recursively define (working from bottom right to top left as in the example depicted below) the 
finite sequences of polynomials $Q_{1}, Q_{2}, \ldots, Q_{r+1}=1$ and $P_{1}=1, P_{2}, \ldots P_{s}$ in $\mathcal{A}_{\beta}[x, y]$ as follows: for $1 \leq i \leq r$,

$$
Q_{i}:=y^{\lambda_{i}-\lambda_{i+1}} Q_{i+1}+\sum_{j=1}^{\lambda_{i+1}} t_{i j} x^{\beta_{j}-i} P_{j},
$$

and for $1 \leq j \leq s$,

$$
P_{j}:=y^{j-\lambda_{\beta_{j}+1}-1} Q_{\beta_{j}+1} .
$$

$$
Q_{1}=y Q_{2}+t_{11} x^{4} P_{1}+t_{12} x^{2} P_{2}+t_{13} x P_{3}+t_{14} x P_{4}
$$

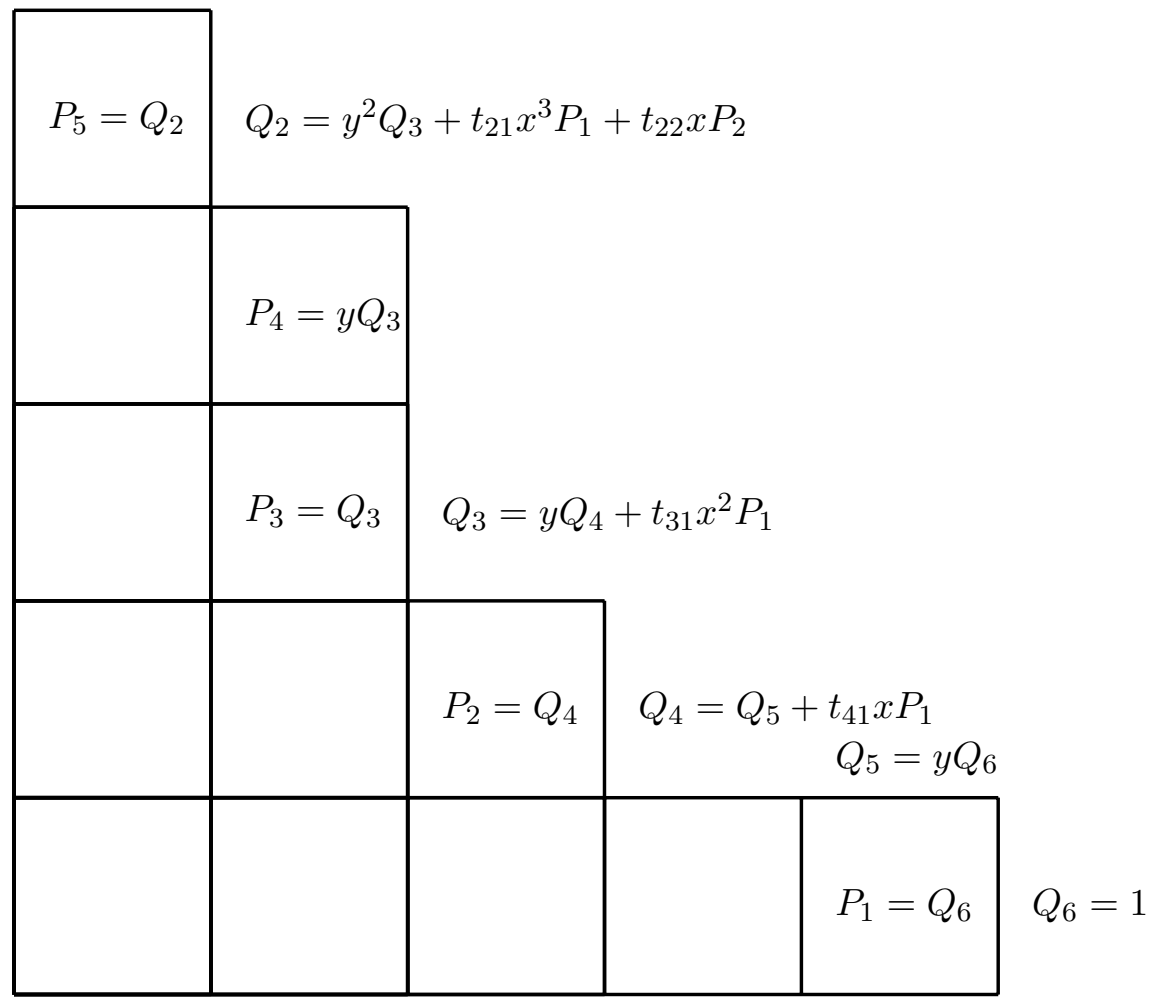

As $\beta_{j} \geq i+1$ when $j \leq \lambda_{i+1}$, by descending induction, for $1 \leq i \leq r+1$

$$
Q_{i} \in y^{\lambda_{i}}+(x),
$$

and by (standard) induction it follows that

$$
P_{i} \in y^{i-1}+(x)
$$

for $1 \leq i \leq s$. For $1 \leq i \leq r+1$, we define

$$
\mathcal{I}_{i}=\left(Q_{i}, x Q_{i+1}, x^{2} Q_{i+2}, \ldots, x^{r+1-i} Q_{r+1}\right) .
$$


Lemma 5.3. For any field $F$ and ring homomorphism $\phi: \mathcal{A}_{\beta} \rightarrow F, I_{1}:=$ $\mathcal{I}_{1} \otimes_{\mathcal{A}_{\beta}, \phi} F$ is an $(x, y)$-primary ideal of $F[x, y]$ of codimension $n$. A linear complement for $I_{1}$ in $F[x, y]$ is given by

$$
\operatorname{Span}\left\{x^{i-1} y^{j} \mid 1 \leq i \leq r, 0 \leq j<\lambda_{i}\right\} .
$$

Moreover, every $(x, y)$-primary ideal of $F[x, y]$ of codimension $n$ satisfying

$$
\operatorname{dim}\left(I_{1}: x^{k}\right) /\left(I_{1}: x^{k-1}\right)=\lambda_{k}, k=1, \ldots, r
$$

arises from one and only one $\phi$.

Proof. Setting $I_{k}:=\mathcal{I}_{k} \otimes_{\mathcal{A}, \phi} F$, we have

$$
I_{k}=\left(\bar{Q}_{k}, x \bar{Q}_{k+1}, \ldots, x^{r+1-k} \bar{Q}_{r+1}\right),
$$

where $\bar{Q}_{k}:=Q_{k} \otimes 1$ belongs to $y^{\lambda_{k}}+(x) \subset F[x, y]$. As

$$
\bar{Q}_{k}=y^{\lambda_{k}-\lambda_{k+1}} \bar{Q}_{k+1}+\sum_{j=1}^{\lambda_{k+1}} a_{k j} x^{\beta_{j}-k} y^{j-\lambda_{\beta_{j}+1}-1} \bar{Q}_{\beta_{j}+1},
$$

where $a_{k j}:=x_{k j} \otimes 1=\phi\left(x_{k j}\right)$, we have

$$
x \bar{Q}_{k} \in\left(x \bar{Q}_{k+1}, \ldots, x^{r+1-k} \bar{Q}_{r+1}\right)=x I_{k+1},
$$

so $R \bar{Q}_{k} \in x I_{k+1}$ if and only if $R \in(x)$. This means an element of $I_{k}$ belongs to $(x)$ if and only if it belongs to $x I_{k+1} \subset I_{k}$, i.e.

$$
\left(I_{k}: x\right)=I_{k+1}
$$

for $1 \leq k \leq r$. By induction, $\left(I_{k}: x^{j}\right)=I_{k+j}$ for $1 \leq k<k+j \leq r+1$. As $I_{r+1}$ is the unit ideal, $x^{r} \in I_{1}$, so the image of $x$ in $F[x, y] / I_{1}$ is nilpotent. As $y^{\lambda_{1}}$ is divisible by $x\left(\bmod I_{1}\right)$, it follows that $y$ is nilpotent in $F[x, y] / I_{1}$. Thus $I_{1}$ is $(x, y)$-primary.

The composition of maps $I_{k} \hookrightarrow F[x, y] \rightarrow F[y]$ sends $x^{i} \bar{Q}_{k+i}$ to 0 for $i>0$ and sends $\bar{Q}_{k}$ to $y^{\lambda_{k}}$. Thus, we have an isomorphism

$$
F[x, y] /\left(I_{k}+(x)\right) \stackrel{\sim}{\rightarrow} F[y] /\left(y^{\lambda_{k}}\right) .
$$

We prove by descending induction that the span of

$$
\left\{x^{i-k} y^{j} \mid k \leq i \leq r, 0 \leq j<\lambda_{i}\right\}
$$

is complementary to $I_{k}$ in $F[x, y]$. This is trivial for $k=r+1$. Multiplication by $x$ gives an isomorphism

$$
F[x, y] / I_{k+1}=F[x, y] /\left(I_{k}: x\right) \rightarrow(x) /\left(I_{k} \cap(x)\right) .
$$

By (5.2), the short exact sequence

$$
0 \rightarrow(x) /\left(I_{k} \cap(x)\right) \rightarrow F[x, y] / I_{k} \rightarrow F[x, y] /\left(I_{k}+(x)\right) \rightarrow 0
$$

can be rewritten

$$
0 \rightarrow F[x, y] / I_{k+1} \rightarrow F[x, y] / I_{k} \rightarrow F[y] /\left(y^{\lambda_{k}}\right) \rightarrow 0 .
$$

By induction,

$$
\operatorname{dim} F[x, y] / I_{k}=\lambda_{k}+\lambda_{k+1}+\cdots+\lambda_{r}
$$


To prove the image of (5.3) spans $F[x, y] / I_{k}$, we assume the corresponding statement for $k+1$. Then

$$
\left\{x^{i-k} y^{j} \mid k+1 \leq i \leq r, 0 \leq j<\lambda_{i}\right\}
$$

spans $(x) /\left(I_{k+1} \cap(x)\right)$ and the image of

$$
\left\{y^{j} \mid 0 \leq j<\lambda_{k}\right\}
$$

spans $F[y] /\left(y^{\lambda_{k}}\right)$, so the image of (5.3) spans $F[x, y] / I_{k}$.

Next we claim that $I_{1}$ determines $\phi$. Equivalently, $I_{1}$ determines $a_{i j}=$ $\phi\left(x_{i j}\right)$. We prove by descending induction that $I_{k}$ determines $a_{i j}$ for all $i \geq k$. This is trivial for $k \geq r+1$. Assume it holds for $k+1$. As $I_{k+1}=\left(I_{k}: x\right)$ determines $a_{i j}$ for $i \geq k+1$ (and therefore determines $\bar{Q}_{k+1}, \ldots, \bar{Q}_{r+1}$ ), we need only consider the case $i=k$. It suffices to prove that

$$
I_{k} \cap \operatorname{Span}\left\{x^{\beta_{j}-k} \bar{P}_{j} \mid 1 \leq j<\lambda_{k+1}\right\}=\{0\} .
$$

Indeed, if

$$
\sum_{j=1}^{\lambda_{k+1}-1} c_{j} x^{\beta_{j}-k} \bar{P}_{j} \in I_{k}
$$

and $m:=\min \left\{\beta_{j} \mid c_{j} \neq 0\right\}$, then this linear combination lies in $I_{k} \cap\left(x^{m-k}\right)=$ $x^{m-k} I_{m}$, and we have

$$
\sum_{j=1}^{\lambda_{m}} c_{j} x^{\beta_{j}-m} \bar{P}_{j} \in I_{m} .
$$

Reducing $(\bmod x)$, we have a non-trivial linear combination of $y^{j-1}$ for $j \leq \lambda_{\beta_{j}} \leq \lambda_{m}$ belonging to $\left(y^{\lambda_{m}}\right)$, which is impossible.

Finally, we claim that every $(x, y)$-primary codimension- $n$ ideal of in $F[x, y]$ can be expressed as $I_{1}$ for some partition $\lambda$ of $n$ and some $\phi$. Defining

$$
\lambda_{i}=\operatorname{dim}\left(I: x^{i}\right) /\left(I: x^{i-1}\right),
$$

we have $\lambda_{1} \geq \lambda_{2} \geq \cdots$ since multiplication by $x$ defines an injection

$$
\left(I: x^{i+1}\right) /\left(I: x^{i}\right) \hookrightarrow\left(I: x^{i}\right) /\left(I: x^{i-1}\right), i \geq 1,
$$

and $\sum_{i=1}^{\infty} \lambda_{i}=n$ since $\left(I: x^{m}\right)=F[x, y]$ for $m$ sufficiently large. This determines $\lambda$, and now we must show that the parameters $a_{i j}$ can be chosen so that $I_{1}=I$. We use induction on the number of parts in the partition.

Given $I$ with associated partition $\lambda_{1} \geq \lambda_{2} \geq \cdots$, let $J:=(I: x)$, which is associated to $\lambda_{2} \geq \lambda_{3} \geq \cdots$. By the induction hypothesis, there exist $a_{i j} \in F$ for $2 \leq i<r, 1 \leq j \leq \lambda_{i+1}$ such that

$$
I_{2}=\left(\bar{Q}_{2}, x \bar{Q}_{3}, \ldots, x^{r-1} \bar{Q}_{r+1}\right)
$$

coincides with $J$. The image of $I$ by the $(\bmod (x))$ reduction map $F[x, y] \rightarrow$ $F[y]$ is $\left(y^{\lambda_{1}}\right)$, so $I=\left(\bar{Q}_{1}\right)+x I_{2}$ for some $\bar{Q}_{1}$ of the form $y^{\lambda_{1}-\lambda_{2}} \bar{Q}_{2}+x \alpha$, where

$$
x \alpha \in(x) \cap J=(x) \cap I_{2}=x I_{3},
$$


i.e., $\alpha \in I_{3}$. On the other hand, if $\alpha-\beta \in I_{2}$, then

$$
\left(y^{\lambda_{1}-\lambda_{2}} \bar{Q}_{2}+x \alpha\right)+x I_{2}=\left(y^{\lambda_{1}-\lambda_{2}} \bar{Q}_{2}+x \beta\right)+x I_{2} .
$$

It suffice to prove that every class in $I_{3} / I_{2}$ is represented by some $\alpha$ of the form $\sum_{i=1}^{\lambda_{2}} a_{1 j} x^{\beta_{j}-2} y^{j-\lambda_{\beta_{j}}-1} \bar{Q}_{\beta_{j}+1}$. Composing the map $F^{\lambda_{2}} \rightarrow I_{3}$ given by

$$
\left(a_{11}, \ldots, a_{1 \lambda_{2}}\right) \mapsto \sum_{j=1}^{\lambda_{2}} a_{1 j} x^{\beta_{j}-2} y^{j-\lambda_{\beta_{j}}-1} \bar{Q}_{\beta_{j}+1} \in I_{3}
$$

with the quotient map $I_{3} \rightarrow I_{3} / I_{2}$, we get an injective map between vector spaces of dimension $\lambda_{2}$, which must therefore be surjective.

Now we can prove Proposition 5.2 ,

Proof. It therefore suffices to prove the equivalent form

$$
\left[R_{n}\right]=\sum_{\lambda \in P(n)}\left[\mathbb{A}^{n-\lambda_{1}}\right]
$$

As $\mathcal{I}_{1}$ contains $(x, y)^{n}$, if $M$ denotes $\mathcal{A}_{\beta}$-module of polynomials of degree $<n$, we have an isomorphism of $\mathcal{A}_{\beta}$-modules $M / M \cap \mathcal{I}_{1} \stackrel{\sim}{\rightarrow} \mathcal{A}_{\beta} / \mathcal{I}_{1}$. The $\mathcal{A}_{\beta}$-linear map

$$
\operatorname{Span}_{\mathcal{A}_{\beta}}\left\{x^{i} y^{j} \mid 0 \leq i<r, 0 \leq j<\lambda_{i+1}\right\} \rightarrow \mathcal{A}_{\beta} / \mathcal{I}_{1}
$$

becomes an isomorphism after tensoring by any residue field of $\mathcal{A}_{\beta}$, so by Nakayama's lemma, it must be an isomorphism. Thus $\mathcal{A}_{\beta}[x, y] / \mathcal{I}_{1}$ is a free $\mathcal{A}_{\beta}$-module, and this remains true after tensoring over $\mathbb{Z}$ with $k$. If $S=$ Spec $\mathcal{A}_{\beta} \otimes_{\mathbb{Z}} k$ and $Z=\operatorname{Spec} \mathcal{A}_{\beta}[x, y] / \mathcal{I}_{1} \otimes_{\mathbb{Z}} k$, then $Z \rightarrow S$ is flat and therefore defines an $S$-point of the Hilbert scheme $\left(\mathbb{A}^{2}\right)^{[n]}$, and since every geometric point of $S$ corresponds to a $(x, y)$-primary ideal, it follows that $S$ maps to $R_{n}$. At the level of $F$-points, this map gives a bijection between $(x, y)$-primary ideals associated to $\lambda$ and $F$-points of $S$. The proposition now follows from the following lemma.

Lemma 5.4. Let $k$ be a field and $\phi: Y \rightarrow X$ a morphism of $k$-varieties. If for all extension fields $F$ of $k, \phi$ defines a bijection from $Y(F)$ to $X(F)$, then $[X]=[Y]$ in $K_{0}\left(\operatorname{Var}_{k}\right)$.

Proof. Suppose $K$ is a field and $Y_{K}$ a $K$-variety such that for every extension field $L$ of $K$, there is a unique morphism Spec $L \rightarrow Y_{K}$ lifting Spec $L \rightarrow$ Spec $K$. If $y_{1}, y_{2}$ are points on $Y_{K}$ with residue fields $K_{1}$ and $K_{2}$ over $K$, we can choose a field $\Omega$ in which $K_{1}$ and $K_{2}$ both embed as subfields, so $Y_{K}$ has at least two distinct $\Omega$-points, contrary to assumption. Thus $Y_{K}$ has a single point, so it is affine: $Y_{K}=$ Spec $A_{K}$ for some reduced $K$-algebra $A_{K}$. The nilradical corresponds to the unique maximal ideal, and it is zero since $Y_{K}$ is a variety, so $A_{K}$ is a field extension of $L$. On the other hand, the identity map Spec $K \rightarrow$ Spec $K$ lifts to Spec $K \rightarrow \operatorname{Spec} A_{K}$, so the extension $K \rightarrow A_{K}$ has an inverse, which means it is trivial. 
We apply this in the case that $K$ is the residue field of the generic point $\eta$ of a component of $X$ and $Y_{K}$ is the fiber of $Y$ over $\eta$. The conclusion is that there exists a point $\eta^{\prime}$ in $Y$ over $\eta$ for which $\phi$ gives an isomorphism of residue fields. Thus, there exist open neighborhoods $U$ of $\eta$ in $X$ and $U^{\prime}$ of $\eta^{\prime}$ in $Y$ such that $\phi^{-1}(U)=U^{\prime}$ and $\phi$ induces an isomorphism $U^{\prime} \rightarrow U$. Replacing $Y$ and $X$ by $Y \backslash U^{\prime}$ and $X \backslash U$ respectively, the restriction of $\phi$ induces a map on $F$-points for all extensions $F$ of $k$, and the lemma follows by Noetherian induction.

\section{Chow motives and finite Galois modules}

Fix a field $k$ and denote by $V(k)$ the category of smooth and projective $k$-varieties and arbitrary morphisms of such varieties. Given $X \in V(k)$ of dimension $d$ we consider the graded Chow ring $A^{*}(X)=\oplus_{r=0}^{d} A^{d-r}(X)$ of cycles on $X$ modulo rational equivalence, where the group $A^{d-r}(X)=A_{r}(X)$ consists of classes of cycles of dimension $r[\mathrm{Fu}$. Let us recall a version of the category of Chow motives that is appropriate for our needs. First consider the additive category $\operatorname{Cor}(k)$ whose objects are the objects of $V(k)$ and morphisms are the degree zero Chow correspondences. That is given $X, Y \in \operatorname{Cor}(k), X$ being of pure dimension $d$, we set

$$
\operatorname{Hom}_{\mathrm{Cor}(k)}(X, Y):=A^{d}(X \times Y)
$$

The composition of morphisms is the composition of correspondences Ma. The category $\operatorname{Cor}(k)$ is the "additivization" of the category $V(k)$. Next one defines the category Chow $(k)$ of Chow motives as the idempotent completion of $\operatorname{Cor}(k)$. Explicitly the objects of Chow $(k)$ are pairs $(X, p)$, where $X \in$ $V(k)$ and $p \in \operatorname{End}_{\operatorname{Cor}(k)}(X)$ is a projector: $p^{2}=p$. Morphisms between $(X, p)$ and $(Y, q)$ form the group $q \cdot \operatorname{Hom}_{\mathrm{Cor}(k)}(X, Y) \cdot p$. There is a canonical contravariant functor $V(k) \rightarrow$ Chow $(k)$ which sends $X \in V(k)$ to $(X, \mathbf{1})$ and a morphism $f: X \rightarrow Y$ to its graph $\Gamma_{f} \subset Y \times X$. Let $e \in \operatorname{Chow}(k)$ be the image of Spec $k$. The category $\operatorname{Chow}(k)$ is a tensor category with the product

$$
(X, p) \otimes(Y, q)=(X \times Y, p \otimes q)
$$

There exists an object $\mathbf{L} \in \operatorname{Chow}(k)$, called the Tate motive, such that $\mathbb{P}^{1}=$ $e \oplus \mathbf{L}[\mathrm{Ma}$. For $(X, p) \in \operatorname{Chow}(k)$ we denote as usual the product $(X, p) \otimes \mathbf{L}$ by $(X, p)(-1)$. Given a nonzero integer $n$ we denote by Chow $(k)[1 / n]$ the localization at $n$ of the additive category $\operatorname{Chow}(k)$, i.e. for $A, B \in \operatorname{Chow}(k)$ we have

$$
\operatorname{Hom}_{\text {Chow }(k)[1 / n]}(A, B)=\operatorname{Hom}_{\text {Chow }(k)}(A, B) \otimes_{\mathbb{Z}} \mathbb{Z}[1 / n]
$$

So Chow $(k)[1 / n]$ is a $\mathbb{Z}[1 / n]$-linear tensor category. We also consider the category $\operatorname{Chow}(k)_{\mathbb{Q}}$ of rational Chow motives constructed in a similar way. 
Example 6.1. Let $X \in V(k)$ be a variety of pure dimension d with an action of a finite group $G$ of order $n$. Then $p:=\frac{1}{n} \sum_{g \in G} \Gamma_{g} \in A^{d}(X \times X) \otimes_{\mathbb{Z}} \mathbb{Z}[1 / n]$ is a projector. Hence $(X, p) \in \operatorname{Chow}(k)[1 / n]$.

Given a field extension $k \subset k^{\prime}$ we obtain the obvious functors $V(k) \rightarrow$ $V\left(k^{\prime}\right)$, Cor $(k) \rightarrow \operatorname{Cor}\left(k^{\prime}\right)$, Chow $(k) \rightarrow$ Chow $\left(k^{\prime}\right)$, etc. induced by the extension of scalars $X \mapsto X_{k^{\prime}}=X \times_{k} k^{\prime}$ of varieties [Fu, Example 6.2.9]. If $k^{\prime}=\bar{k}$, as usual, we denote the variety $X \times_{k} \bar{k}$ by $\bar{X}$. For a prime $l \neq \operatorname{char}(k)$ let $\zeta_{l} \in \bar{k}$ be an $l$-th root of 1 .

Proposition 6.2. Let $n$ be a nonzero integer and $l$ be a prime number not dividing $n$ and different from the characteristic of the base field $k$. Then the assignment

$$
X \mapsto H^{\bullet}\left(\bar{X}, \overline{\mathbb{F}}_{l}\right), \quad X \in V(k)
$$

extends to a tensor (contravariant) functor from the category Chow $(k)[1 / n]$ to the abelian tensor category of finite dimensional $\overline{\mathbb{F}}_{l}$-modules with a continuous $\mathrm{Gal}_{k}$-action:

$$
\Phi_{l}: \operatorname{Chow}(k)[1 / n] \rightarrow \overline{\mathbb{F}}_{l}-\mathrm{Gal}_{k}-\bmod
$$

If $k$ contains $\zeta_{l}$, then the module $\Phi_{l}(\mathbf{L})$ is a 1-dimensional trivial $\overline{\mathbb{F}}_{l}$-Gal ${ }_{k}$ module placed in degree 2.

We do not claim originality for this proposition, but for lack of a reference, we provide a proof.

Proof. Since the category $\overline{\mathbb{F}}_{l}-\mathrm{Gal}_{k}$-mod is Karoubian and its localization $\left(\overline{\mathbb{F}}_{l}-\mathrm{Gal}_{k}\right.$-mod $)[1 / n]$ is equivalent to $\overline{\mathbb{F}}_{l}$-Gal $k_{k}$-mod, it suffices to construct a functor from the additive category $\operatorname{Cor}(k)$ to $\overline{\mathbb{F}}_{l}$-Gal ${ }_{k}$-mod. We construct this functor as the composition of the extension of scalars functor $\operatorname{Cor}(k) \rightarrow$ $\operatorname{Cor}(\bar{k})$ with a functor

$$
\Psi_{l}: \operatorname{Cor}(\bar{k}) \rightarrow \overline{\mathbb{F}}_{l} \text {-vect }
$$

where $\overline{\mathbb{F}}_{l}$-vect is the category of $\overline{\mathbb{F}}_{l}$-vector spaces. The functor $\Psi_{l}$ is defined as follows. Let $X$ and $Y$ be smooth projective varieties (over $\bar{k}$ ), $X$ being of pure dimension $d$, and let $C \in A^{d}(X \times Y)$ be a correspondence of degree zero. Consider the projections $X \stackrel{p_{X}}{\leftarrow} X \times Y \stackrel{p_{Y}}{\rightarrow} Y$. Then given an element $a \in H^{i}\left(Y, \overline{\mathbb{F}}_{l}\right)$ we put

$$
\Psi_{l}(C)(a)=p_{X *}\left(\operatorname{cl}_{X \times Y}(C) \cup p_{Y}^{*}(a)\right) \in H^{i}\left(X, \overline{\mathbb{F}}_{l}\right)
$$

where $\operatorname{cl}_{X \times Y}: A^{s}(X \times Y) \rightarrow H^{2 s}\left(X \times Y, \overline{\mathbb{F}}_{l}\right)$ is the cycle map [SGA $4 \frac{1}{2}$ ], [Mi, Ch.VI, 9], and $p_{Y}^{*}$ and $p_{X *}$ are the pullback and the pushforward maps on cohomology [Mi, Ch. VI, Remark 11.6]. In order for $\Psi_{l}$ to be a functor, the cycle map has to satisfy the following properties for morphisms of smooth and projective varieties:

- $\mathrm{cl}$ is a morphism of contravariant functors from $V(\bar{k})$ to the category of rings.

- cl commutes with exterior products. 
- $\mathrm{cl}$ is a morphism of covariant functors from $V(\bar{k})$ to the category of abelian groups.

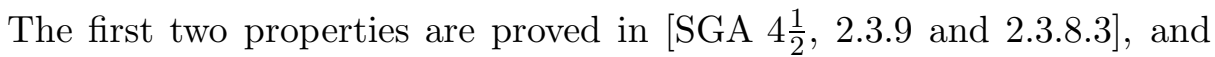
the last one is in [Lau, Theorem 6.1].

Once the functor $\Psi_{l}$ is constructed, it is clear that its composition with the extension of scalars $\operatorname{Cor}(k) \rightarrow \operatorname{Cor}(\bar{k})$ will give the desired functor $\Phi_{l}$, since for $X \in \operatorname{Cor}(k)$ the vector space $H^{\bullet}\left(\bar{X}, \overline{\mathbb{F}}_{l}\right)$ is a $\mathrm{Gal}_{k}$-module and morphisms in $\operatorname{Cor}(k)$ act as morphisms of $\mathrm{Gal}_{k}$-modules. Also the last assertion of the proposition is obvious. This proves Proposition 6.2.

Example 6.3. Let $(X, p) \in \operatorname{Chow}(k)[1 / n]$ be as in Example 6.1 and let $l$ be prime to $n$ and $l \neq \operatorname{char}(k)$. Then $\Phi_{l}((X, p))=H^{\bullet}\left(\bar{X}, \overline{\mathbb{F}}_{l}\right)^{G}$ as $\overline{\mathbb{F}}_{l}-\mathrm{Gal}_{k}$ modules.

Corollary 6.4. Assume that in Chow $(k)_{\mathbb{Q}}$ we have an isomorphism of objects $A \simeq B$. Then for a divisible enough integer $n$ the objects $A$ and $B$ belong to the essential image of the category Chow $(k)[1 / n]$ and are isomorphic in $\operatorname{Chow}(k)[1 / n]$. Fix one such $n$ and let $l$ be a prime not dividing $n$ and $l \neq \operatorname{char}(k)$. Then the $\overline{\mathbb{F}}_{l}$-Gal $\mathrm{Ga}_{k}$-modules $\Phi_{l}(A)$ and $\Phi_{l}(B)$ are defined and are isomorphic.

Proof. Indeed, an isomorphism in $\operatorname{Chow}(k)_{\mathbb{Q}}$ between $A$ and $B$ is witnessed by a finite diagram of objects and correspondences with denominators. Hence this diagram exists in $\operatorname{Chow}(k)[1 / n]$ for a divisible enough $n$. So $A \simeq B$ in such category $\operatorname{Chow}(k)[1 / n]$. The last assertion now follows from Proposition 6.2.

The category Chow $(k)_{\mathbb{Q}}$ can be extended to include objects $\left(X^{\prime}, p^{\prime}\right)$ where $X^{\prime}$ is a quotient variety under the action of a finite group on a smooth projective variety, and $p^{\prime} \in A\left(X^{\prime} \times X^{\prime}\right)_{\mathbb{Q}}$ is a projector [Fu, Example 16.1.13]. Denote the resulting category by $\operatorname{Chow}(k)_{\mathbb{Q}}^{\prime}$. The following lemma is proved in $\mathrm{dBN}, 1.2]$.

Lemma 6.5. 1) The obvious functor $\operatorname{Chow}(k)_{\mathbb{Q}} \rightarrow \operatorname{Chow}(k)_{\mathbb{Q}}^{\prime}$ is an equivalence of categories.

2) Let $X$ be a smooth projective variety with an action of a finite group $G$. Consider the motive $(X, p) \in$ Chow $(k)_{\mathbb{Q}}$ as in Example 6.1. Then the motives $(X, p)$ and $(X / G, \mathbf{1}) \in \operatorname{Chow}(k)_{\mathbb{Q}}^{\prime}$ are isomorphic.

We remark that the measures $\mu_{l}$ and $\nu_{l}$ defined in $\S 4$ factor through $K_{0}(\operatorname{Chow}(k)[1 / n])$ if $l \nmid n$. Indeed, by a theorem of Gillet and Soulé GS, Theorem 4], the correspondence $X \mapsto(X, \mathbf{1})$ for a smooth and projective $X$ extends to a group homomorphism $\theta: K_{0}\left(\operatorname{Var}_{k}\right) \rightarrow K_{0}($ Chow $(k))$, where $K_{0}($ Chow $(k))$ is the Grothendieck group of the additive category Chow $(k)$. Denote by $\theta[1 / n]$ the composition of $\theta$ with the obvious homomorphism $K_{0}(\operatorname{Chow}(k)) \rightarrow K_{0}(\operatorname{Chow}(k))[1 / n]$. 
Assume that the base field $k$ is a subfield of $\mathbb{C}$. The additive functor $\Phi_{l}$ of Proposition 6.2 induces the group homomorphism

$$
K_{0}\left(\Phi_{l}\right): K_{0}(\operatorname{Chow}(k)[1 / n]) \rightarrow K_{l}^{\mathrm{sp}}\left(\overline{\mathbb{F}}_{l}-\mathrm{Gal}_{k}\right)
$$

such that we have the equality

$$
\mu_{l}=K_{0}\left(\Phi_{l}\right) \circ \theta[1 / n]: K_{0}\left(\operatorname{Var}_{k}\right) \rightarrow K_{l}^{\mathrm{sp}}\left(\overline{\mathbb{F}}_{l}-\mathrm{Gal}_{k}\right)
$$

and hence also

$$
\nu_{l}=\operatorname{Res}_{\mathrm{Gal}_{k\left(\zeta_{l}\right)}}^{\mathrm{Gal}_{k}} \circ K_{0}\left(\Phi_{l}\right) \circ \theta[1 / n]: K_{0}\left(\operatorname{Var}_{k}\right) \rightarrow K_{l}^{\mathrm{sp}}\left(\overline{\mathbb{F}}_{l}-\operatorname{Gal}_{k\left(\zeta_{l}\right)}\right)
$$

We obtain the following important corollary which is used in the proof of our main Theorem 7.1 below.

Corollary 6.6. Assume that $k \subset \mathbb{C}$ and let $X$ be a smooth projective variety over $k$ with an action of a finite group $G$. Then for all primes $l$ sufficiently large in terms of $|G|$ we have

$$
\mu_{l}([X / G])=\left[H^{\bullet}\left(\bar{X}, \overline{\mathbb{F}}_{l}\right)^{G}\right] \in K_{l}^{\mathrm{sp}}\left(\overline{\mathbb{F}}_{l}-\mathrm{Gal}_{k}\right)
$$

and hence also

$$
\nu_{l}([X / G])=\left[H^{\bullet}\left(\bar{X}, \overline{\mathbb{F}}_{l}\right)^{G}\right] \in K_{l}^{\mathrm{sp}}\left(\overline{\mathbb{F}}_{l}-\mathrm{Gal}_{k\left(\zeta_{l}\right)}\right)
$$

Proof. By 6.1 it suffices to prove that for some $m$ and all $l$ sufficiently large in terms of $|G|$ we have

$$
K_{0}\left(\Phi_{l}\right) \circ \theta[1 / m]([X / G])=\left[H^{\bullet}\left(\bar{X}, \overline{\mathbb{F}}_{l}\right)^{G}\right] \in K_{l}^{\mathrm{sp}}\left(\overline{\mathbb{F}}_{l}-\mathrm{Gal}_{k}\right)
$$

Let $|G|=n$ and let $(X, p) \in \operatorname{Chow}(k)[1 / n]$ be as in Example6.1. By Lemma 6.5 there is an isomorphism of objects $(X / G, \mathbf{1}) \simeq(X, p)$ in the equivalent categories $\operatorname{Chow}^{\prime}(k)_{\mathbb{Q}} \simeq \operatorname{Chow}(k)_{\mathbb{Q}}$. Hence the same isomorphism holds in Chow $(k)[1 / m]$ for a divisible enough $m$ (Corollary 6.4). Fix one such $m$ and let $l$ be a prime not dividing $m$. Then

$$
\begin{aligned}
\mu_{l}([X / G])=K_{0}\left(\Phi_{l}\right) \circ \theta[1 / m]([X / G]) & =K_{0}\left(\Phi_{l}\right)([(X, p)]) \\
& =\left[\Phi_{l}((X, p))\right]=\left[H^{\bullet}\left(\bar{X}, \overline{\mathbb{F}}_{l}\right)^{G}\right]
\end{aligned}
$$

where the last equality is by Example 6.3. This proves the corollary.

\section{THE MAIN THEOREM}

In this section, we prove the main result of this paper:

Theorem 7.1. There exists a $K 3$ surface $X / \mathbb{Q}$ such that

$$
\zeta_{X}(t) \in K_{0}\left[\operatorname{Var}_{\mathbb{Q}}\right][1 / t][[t]]
$$

is irrational in the sense that if $B(t)$ is a polynomial with coefficients in $K_{0}\left[\operatorname{Var}_{\mathbb{Q}}\right][1 / E][t]$ and $B(0)=1$, then $B(t) \zeta_{X}(t)$ is not a polynomial. 
Proof. Choose $X$ to be the variety denoted $X^{\prime}$ in Proposition 4.4. As usual we let $X^{[i]}$ denote the $i$ th Hilbert scheme of points on $X$. We assume the theorem is not true and choose $B(t)$ with $B(0)=1$ such that $A(t):=$ $B(t) \zeta_{X}(t)$ is a polynomial. Let $n=\operatorname{deg} A+\operatorname{deg} B+24$.

We fix a prime $l$ sufficiently large that:

(1) The homomorphism (4.1) is an isomorphism, i.e., $\operatorname{Gal}\left(K_{1} K_{2} / \mathbb{Q}\left(\zeta_{l}\right)\right)$ is isomorphic to $\mathrm{SL}_{2}\left(\mathbb{F}_{l}\right)^{2}$.

(2) For all $i \leq n$, we have $\nu_{l}\left(\left[\operatorname{Sym}^{i} X\right]\right)=\left[\operatorname{Sym}^{i} H^{\bullet}\left(\bar{X}^{n}, \overline{\mathbb{F}}_{l}\right)\right]$.

For large enough $l,(1)$ holds by Theorem 4.4, and (2) holds by Corollary 4.3 and Corollary 6.6,

We set $G:=\mathrm{SL}_{2}\left(\mathbb{F}_{l}\right)^{2}$, which we identify with $\operatorname{Gal}\left(K_{1} K_{2} / \mathbb{Q}\left(\zeta_{l}\right)\right)$. Thus, for $1 \leq i \leq n$, we have

$$
a_{i}:=\nu_{l}\left(\left[\operatorname{Sym}^{i} X\right]\right)=\operatorname{Res}_{G_{\mathbb{Q}\left(\zeta_{l}\right)}^{G}}^{G}\left[\operatorname{Sym}^{i}\left(\overline{\mathbb{F}}_{l}^{20} \oplus V_{1} \otimes V_{1}\right)\right] \in \operatorname{Res}_{G_{\mathbb{Q}\left(\zeta_{l}\right)}^{G}} K_{l}^{\mathrm{sp}}(G) .
$$

Let $b_{i}:=\nu_{l}\left(\left[X^{[i]}\right]\right)$ for $i \geq 0$. Thus $b_{i}$ is effective for all $i$, and by Theorem $[5.1$. $a_{i}$ and $b_{i}$ satisfy the identities given by (2.10).

Applying Lemma 2.6 with $R=K_{l}^{\mathrm{sp}}\left(G_{\mathbb{Q}\left(\zeta_{l}\right)}\right)$, which contains $K_{l}^{\mathrm{sp}}(G)$ via the map $\operatorname{Res}_{G_{\mathbb{Q}\left(\zeta_{l}\right)}^{G}}$, we deduce that $\nu_{l}\left(X^{[l]}\right)$ is not effective, which is a contradiction.

\section{REFERENCES}

[AJL] Andersen, Henning Haahr; Jørgensen, Jens; Landrock, Peter: The projective indecomposable modules of $\mathrm{SL}\left(2, p^{n}\right)$. Proc. London Math. Soc. (3) 46 (1983), no. $1,38-52$.

[Ay1] Ayoub, Joseph: Topologie feuilletée et la théorie de Galois différentielle, preprint, 2017, http://user.math.uzh.ch/ayoub/PDF-Files/Feui-DGal.pdf

[Ay2] Ayoub, Joseph: Topologie feuilletée et la conservativité des réalisations classiques en caractéristique nulle, preprint, 2018, http://user.math.uzh.ch/ayoub/PDF-Files/Feui-ConCon.pdf

[dBN] del Baño Rollin, Sebastian; Navarro Aznar, Vicente: On the motive of a quotient variety. Collect. Math. 49 (1998), no. 2-3, 203-226.

[Bi] Bittner, Franziska: The universal Euler characteristic for varieties of characteristic zero. Compos. Math. 140 (2004), no. 4, 1011-1032.

[Bon] Bondarko, Mikhail: Conservativity of realizations implies that numerical motives are Kimura-finite and motivic zeta functions are rational, arXiv:1807.10791

[Bor] Borisov, Lev: Class of the affine line is a zero divisor in the Grothendieck ring, preprint, arXiv:1412.6194 3 .

[SGA 4 $\frac{1}{2}$ ] Deligne, Pierre: Cohomologie étale. Séminaire de Géométrie Algébrique du Bois-Marie SGA $4 \frac{1}{2}$. Avec la collaboration de J. F. Boutot, A. Grothendieck, L. Illusie et J. L. Verdier. Lecture Notes in Mathematics, Vol. 569.

[SGA 7] Deligne, Pierre; Katz, Nicholas: Groupes de monodromie en géométrie algébrique. II. Séminaire de Géométrie Algébrique du Bois-Marie 1967-1969 (SGA 7 II). Lecture Notes in Mathematics, Vol. 340. Springer-Verlag, BerlinNew York, 1973. 
[DL] Denef, Jan; Loeser, François: On some rational generating series occurring in arithmetic geometry. Geometric aspects of Dwork theory. Vol. I, II, 509526, Walter de Gruyter, Berlin, 2004.

[Dw] Dwork, Bernard: On the rationality of the zeta function of an algebraic variety. Amer. J. Math. 82 (1960), 631-648.

[ES] Ellingsrud, Geir; Strømme, Stein Arild: On the homology of the Hilbert scheme of points in the plane. Invent. Math. 87 (1987), no. 2, 343-352.

[Fu] Fulton, William: Intersection theory. Ergebnisse der Mathematik und ihrer Grenzgebiete (3), 2. Springer-Verlag, Berlin, 1984.

[GS] Gillet, Henri; Soulé, Christophe: Descent, motives and K-theory. J. Reine Angew. Math. 478 (1996), 127-176.

[Go] Göttsche, Lothar: On the motive of the Hilbert scheme of points on a complex surface. Math. Res. Lett. 8 (2001), 613-627.

[Gr] Grothendieck, Alexandre: Sur quelques points d'algèbre homologique. Tôhoku Mathematical Journal (2) 9 (1957), 119-221.

[Ka] Kapranov, Mikhail: The elliptic curve in the S-duality theory and Eisenstein series for Kac-Moody groups, arXiv: math.AG/0001005.

[Kr] Krause, Henning: Krull-Schmidt Categories and Projective Covers, arXiv:1410.2822.

[LL] Larsen, Michael; Lunts, Valery A.: Motivic measures and stable birational geometry. Mosc. Math. J. 3 (2003), no. 1, 85-95.

[LL2] Larsen, Michael; Lunts, Valery A.: Rationality criteria for motivic zeta functions. Compos. Math. 140 (2004), no. 6, 1537-1560.

[Lau] Laumon, Gérard: Homologie étale. Séminaire de géométrie analytique (École Norm. Sup., Paris, 1974-75), pp. 163-188. Astérisque, No. 36-37, Soc. Math. France, Paris, 1976.

[Law] Lawrence, John: When is the tensor product of algebras local? II. Proc. Amer. Math. Soc. 58 (1976), 22-24.

[Ma] Manin, Ju. I.: Correspondences, motifs and monoidal transformations. (Russian) Mat. Sb. (N.S.) 77 (119) 1968, 475-507.

[Mi] Milne, James S.: Étale cohomology. Princeton Mathematical Series, 33. Princeton University Press, Princeton, N.J., 1980.

[Po] Poonen, Bjorn: The Grothendieck ring of varieties is not a domain. Math. Res. Lett. 9 (2002), no. 4, 493-497.

[Se] Serre, Jean-Pierre: Propriétés galoisiennes des points d'ordre fini des courbes elliptiques. Invent. Math. 15 (1972), no. 4, 259-331.

[Si] Silverman, Joseph H.: Advanced topics in the arithmetic of elliptic curves. Graduate Texts in Mathematics, 151. Springer-Verlag, New York, 1994.

[Za] Zakharevich, Inna: The annihilator of the Lefschetz motive. Duke Math. J. 166 (2017), no. 11, 1989-2022.

Department of Mathematics, Indiana University, Bloomington, IN 47405, U.S.A.

Department of Mathematics, Indiana University, Bloomington, IN 47405, U.S.A. 